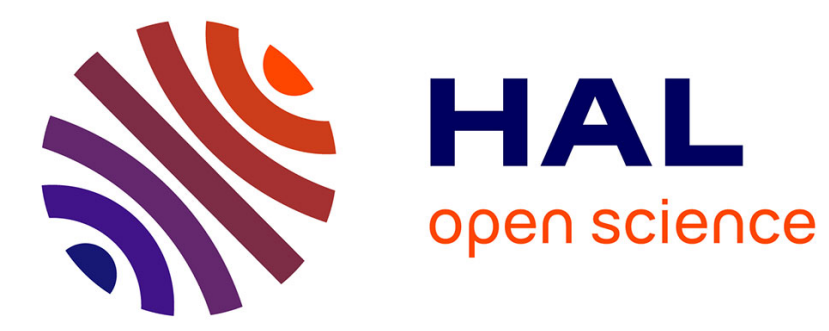

\title{
Stability of a Crank-Nicolson Pressure Correction Scheme Based on Staggered Discretizations
}

Franck Boyer, Fanny Dardalhon, Céline Lapuerta, Jean-Claude Latché

\section{To cite this version:}

Franck Boyer, Fanny Dardalhon, Céline Lapuerta, Jean-Claude Latché. Stability of a Crank-Nicolson Pressure Correction Scheme Based on Staggered Discretizations. International Journal for Numerical Methods in Fluids, 2013, 74 (1), pp.34-58. 10.1002/fld.3837 . hal-00721597v2

\section{HAL Id: hal-00721597 \\ https://hal.science/hal-00721597v2}

Submitted on 3 May 2013

HAL is a multi-disciplinary open access archive for the deposit and dissemination of scientific research documents, whether they are published or not. The documents may come from teaching and research institutions in France or abroad, or from public or private research centers.
L'archive ouverte pluridisciplinaire HAL, est destinée au dépôt et à la diffusion de documents scientifiques de niveau recherche, publiés ou non, émanant des établissements d'enseignement et de recherche français ou étrangers, des laboratoires publics ou privés. 


\title{
Stability of a Crank-Nicolson Pressure Correction Scheme Based on Staggered Discretizations
}

\author{
F. Boyer ${ }^{1}$, F. Dardalhon ${ }^{1,2}$, C. Lapuerta ${ }^{2}$, J.-C. Latché ${ }^{2}$ \\ ${ }^{1}$ Aix-Marseille Université, Laboratoire d'Analyse, Topologie et Probabilités, \\ Centre de Mathématiques et Informatique, 39 rue Joliot-Curie \\ 13453 Marseille Cedex 13, France. \\ ${ }^{2}$ Institut de Radioprotection et de Sûreté Nucléaire (IRSN) \\ BP3 - 13115 Saint Paul-lez-Durance CEDEX, France. \\ fboyerelatp.univ-mrs.fr, fanny.Dardalhonelatp.univ-mrs.fr, \\ celine.lapuertalirsn.fr, jean-claude.latchelirsn.fr
}

\section{SUMMARY}

In the context of Large Eddy Simulation of turbulent flows, the control of kinetic energy seems to be an essential requirement for a numerical scheme. Designing such an algorithm, i.e. as less dissipative as possible while being simple, for the resolution of variable density Navier-Stokes equations is the aim of the present work. The developed numerical scheme, based on a pressure correction technique, uses a CrankNicolson time discretization and a staggered space discretization relying on the Rannacher-Turek finite element. For the inertia term in the momentum balance equation, we propose a finite volume discretization, for which we derive a discrete analogue of the continuous kinetic energy local conservation identity. Contrary to what was obtained for the backward Euler discretization, the dissipation defect term associated to the Crank-Nicolson scheme is second order in time. This behaviour is evidenced by numerical simulations.

KEY WORDS: Pressure correction scheme, compressible Navier-Stokes equations, stable convection operator, low-order finite element approximation

\section{CONTENTS}

1 Introduction 2

2 Meshes and discrete spaces $\quad 4$

3 Construction of a stable convection operator 5

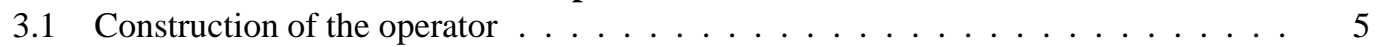

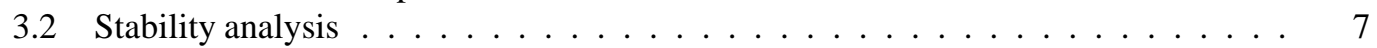

4 A Crank-Nicolson-like pressure correction scheme 9

4.1 The time-marching algorithm $\ldots \ldots \ldots \ldots$

4.2 The fully discrete scheme . . . . . . . . . . . . . . . . . . . 11

4.3 The discrete kinetic energy identity . . . . . . . . . . . . . . . . . . . . . 12

4.4 Order in time of the dissipation defect . . . . . . . . . . . . . . . . . . . . . 14

5 Numerical experiments 15

5.1 Convergence and dissipation defect $\ldots \ldots \ldots \ldots \ldots \ldots \ldots$

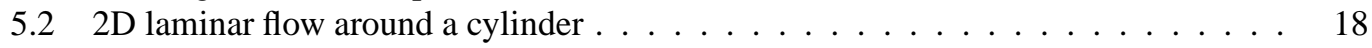

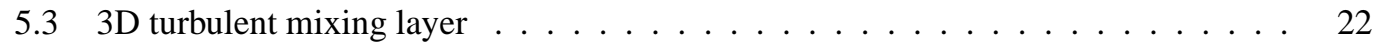

*Correspondence to: fboyer@latp.univ-mrs.fr 


\section{INTRODUCTION}

We consider the time-dependent variable density Navier-Stokes equations, posed on a finite time interval $(0, T)$ and in an open, connected, bounded domain $\Omega$ of $\mathbb{R}^{d}(d=2$ or 3$)$, which is supposed to be polygonal $(d=2)$ or polyhedral $(d=3)$ :

$$
\mid \begin{aligned}
& \partial_{t} \rho+\operatorname{div}(\rho \boldsymbol{u})=0, \\
& \partial_{t}(\rho \boldsymbol{u})+\operatorname{div}(\boldsymbol{u} \otimes \rho \boldsymbol{u})-\operatorname{div}(\tau(\boldsymbol{u}))+\nabla p=0,
\end{aligned}
$$

where $\boldsymbol{u}, p$ and $\rho$ are respectively the velocity, the pressure and the density of the flow. The quantity

$$
\tau(\boldsymbol{u})=\mu\left[\boldsymbol{\nabla} \boldsymbol{u}+\nabla^{T} \boldsymbol{u}-\frac{2}{3}(\operatorname{div} \boldsymbol{u}) I\right]
$$

is the shear stress tensor, with $\mu$ the positive kinematic viscosity, and $\boldsymbol{u} \otimes \rho \boldsymbol{u}$ is the $\mathbb{R}^{d} \times \mathbb{R}^{d}$-tensor of components $(\boldsymbol{u} \otimes \rho \boldsymbol{u})_{i, j}=\rho \boldsymbol{u}_{i} \boldsymbol{u}_{j}, \forall i, j \in\{1, \cdots, d\}$. As such, System (1) is not closed. The simplest assumption yielding a self-contained problem is just supposing that $\rho$ is a given function of space and time; the mass balance equation (i.e. the first relation of (1)) thus has to be considered as a constraint on the velocity, as for incompressible flows (and, indeed, choosing a constant value for $\rho$ yields $\operatorname{div} \boldsymbol{u}=0)$. This equation basically plays the same role in the so-called asymptotic model for low Mach number flows [19], where $\rho$ is given as a function of an additional unknown $\theta$ (usually, the temperature or a concentration), which satisfies a balance equation:

$$
\partial_{t}(\rho \theta)+\operatorname{div}(\rho \theta \boldsymbol{u})-\operatorname{div}(\lambda \nabla \theta)=0, \quad \rho=\varrho(\theta) .
$$

The function $\theta \mapsto \varrho(\theta)$ is the given equation of state for the flow under study, and $\lambda$ is a non-negative diffusion coefficient.

System (1)-(2) must be supplemented by suitable boundary conditions, for instance Dirichlet conditions for $\boldsymbol{u}$ and $\theta$, or slip and Neumann conditions:

$$
\boldsymbol{u} \cdot \boldsymbol{n}=0, \quad((\tau(\boldsymbol{u})-p I) \boldsymbol{n}) \cdot \boldsymbol{t}=0, \quad \text { and } \quad \boldsymbol{\nabla} \theta \cdot \boldsymbol{n}=0 \text { on }(0, T) \times \partial \Omega,
$$

where $n$ and $t$ are respectively the outward unit normal and tangential vectors to $\partial \Omega$. Initial conditions are given for $\boldsymbol{u}$ and $\theta$, namely $\boldsymbol{u}=\boldsymbol{u}_{0}$ and $\theta=\theta_{0}$ in $\Omega$. We suppose that the equation of state satisfies $\varrho(\theta)>0$ for $\theta>0$, that $\theta_{0}>0$, and that boundary conditions are such that $\theta$ remains positive for all $t \in(0, T)$.

The density $\rho$ and the velocity $\boldsymbol{u}$ are known to satisfy the so-called kinetic energy identity. This relation stems from the following formal computation.

Property 1.1 (Property K.E.)

Let us assume that

$$
\partial_{t} \rho+\operatorname{div}(\rho \boldsymbol{\beta})=0
$$

for a regular field $\boldsymbol{\beta}$ and that the functions $\rho$ and $\boldsymbol{u}$ are smooth. Then, we have:

$$
\left(\partial_{t}(\rho \boldsymbol{u})+\operatorname{div}(\boldsymbol{u} \otimes \rho \boldsymbol{\beta})\right) \cdot \boldsymbol{u}=\frac{1}{2} \partial_{t}\left(\rho|\boldsymbol{u}|^{2}\right)+\frac{1}{2} \operatorname{div}\left(\rho|\boldsymbol{u}|^{2} \boldsymbol{\beta}\right) .
$$

Integrating this relation over $\Omega$ yields, assuming that $\boldsymbol{\beta} \cdot \boldsymbol{n}=0$ on $\partial \Omega$ :

$$
\int_{\Omega}\left(\partial_{t}(\rho \boldsymbol{u})+\operatorname{div}(\boldsymbol{u} \otimes \rho \boldsymbol{\beta})\right) \cdot \boldsymbol{u} \mathrm{d} \boldsymbol{x}=\frac{1}{2} \frac{d}{d t} \int_{\Omega} \rho|\boldsymbol{u}|^{2} \mathrm{~d} \boldsymbol{x} .
$$

Applying identity (4), with $\boldsymbol{\beta}=\boldsymbol{u}$, to the inner product of the momentum balance equation (second relation of (1)) with $\boldsymbol{u}$ yields the so-called (local) kinetic energy identity:

$$
\frac{1}{2} \partial_{t}\left(\rho|\boldsymbol{u}|^{2}\right)+\frac{1}{2} \operatorname{div}\left(\rho|\boldsymbol{u}|^{2} \boldsymbol{u}\right)+\boldsymbol{u} \cdot \nabla p-\operatorname{div}(\tau(\boldsymbol{u})) \cdot \boldsymbol{u}=0 .
$$


The first two terms of this relation are the time derivative and the transport term of the kinetic energy, respectively; the third one corresponds to the power of the pressure forces, and the last term of the left-hand side reads $-\operatorname{div}(\tau(\boldsymbol{u})) \cdot \boldsymbol{u}=-\operatorname{div}(\tau(\boldsymbol{u}) \boldsymbol{u})+\tau(\boldsymbol{u}): \nabla \boldsymbol{u}$, which makes appear the so-called viscous dissipation $\tau(\boldsymbol{u}): \boldsymbol{\nabla} \boldsymbol{u}$. Integrating over $\Omega$, then integrating by parts the viscous term and using the boundary conditions, we obtain the (global) kinetic energy conservation relation:

$$
\frac{1}{2} \frac{d}{d t} \int_{\Omega} \rho|\boldsymbol{u}|^{2} \mathrm{~d} \boldsymbol{x}+\int_{\Omega} \boldsymbol{u} \cdot \boldsymbol{\nabla} p \mathrm{~d} \boldsymbol{x}+\int_{\Omega} \tau(\boldsymbol{u}): \nabla \boldsymbol{u} \mathrm{d} \boldsymbol{x}=0 .
$$

This relation does not yield, in its present form, a stability estimate, because of the presence of the pressure term. For incompressible flows, a simple integration by parts shows that it vanishes; for barotropic compressible flows, it may be recast as the time derivative of an energy term. For the low Mach number model, the correct way to treat this term is unclear.

Obtaining a scheme satisfying a discrete analogue of (6)-(7) has many advantages.

- First, possibly combined with the above-mentioned additional arguments to control the pressure term, (7) yields a stability estimate, which is classically observed to considerably enhance the robustness of the scheme, especially for the computation of convection dominant flows.

- Second, in the context of Large Eddy Simulation (LES), a subgrid-scale model is introduced to simulate the (kinetic energy) dissipative role of the small structures in the momentum balance equation. It is thus crucial that the scheme respects this "physical" dissipation, i.e. does not overwhelm the viscous dissipation term in (the discrete counterpart of) Equation (6) by too large numerical residual terms [21, 2]. Defining these latter as the "dissipation defect", when designing a numerical scheme for LES, one aim should be to minimize the "dissipation defect".

The Marker And Cell (MAC) space discretization, first introduced in [13] and now widely used for the simulation of incompressible flows, applies to Cartesian meshes and is of staggered type, with the pressure unknowns located at the cell centers and the velocity normal components unknowns at the face centers. For divergence-free constant density flows, it has been observed since the middle of the sixties that the natural discrete convection operator associated to this discretization conserves the discrete kinetic energy [18], i.e. satisfies a discrete analogue of the integral identity (5) (of course, supposing, in this latter, a constant density $\rho$ ). Higher order convection operators, still enjoying the same property, and also a discrete analogue of the local kinetic energy transport equation (5), have been proposed in $[23,32,33,31]$; combined with a suitable discretization of the pressure gradient term and a suitable time-marching algorithm, these results yield (discrete analogues to) the local and global kinetic energy balances (6) and (7).

Works concerning the MAC scheme for the compressible low Mach number model are more recent and scarcer. First, [24] generalized the finite difference formula of [23], to obtain a scheme which appears, from numerical experiments, to satisfy a discrete version of the global identity (7) up to a second order residual. A similar approach, adapted to cope with cylindrical coordinates, is proposed in [5]. Recently, a (structured) MAC scheme is presented in [22], followed by a discussion about its conservation properties: both global and local kinetic energy conservation are shown, in both semi-discrete and fully-discrete settings. However, the resulting scheme seems to be rather expensive, and more efficient alternatives with nonzero but still high order residuals are also proposed. For related works in the collocated framework, see $[6,8,12,10]$.

In this article, we pursue the development of kinetic energy conserving schemes for low Mach number flows, with the aim to deal with unstructured staggered discretizations. Precisely speaking, we build a discrete convection operator satisfying the control of the kinetic energy and the reduction of the dissipation defect (discrete analogues of (4) and (5)) with high-order numerical residuals. The approach we adopt is based on a finite volume structure for the convection operator first introduced in [1]; the approximation of the convected velocity at the face is centered, and the time-algorithm is obtained by a Crank-Nicolson technique. Then we implement this discretization in a pressure correction scheme, and make the expression of the dissipation defect explicit. This quantity appears to be formally second order in time, but is unsigned (unlike the first order counterpart which would 
arise from a backward Euler discretization). It is plotted in numerical experiments to assess the performance of the scheme.

The outline of the article is as follows. After introducing the meshes and the discrete spaces in Section 2, we deal in Section 3 with the construction of the convection operator (Section 3.1) and prove its stability (Property K.E., Section 3.2). The pressure correction scheme for low Mach number flows is presented in Section 4, first in the semi-discrete setting, then in the fully discrete one (Sections 4.1 and 4.2 respectively). The kinetic energy identity satisfied by the fully discrete scheme is established (Section 4.3), then the size of the dissipation defect is evaluated (Section 4.4). Numerical tests are presented in Section 5; they confirm theoretical results and illustrate the ability of the scheme to compute quite efficiently turbulent structures for large time steps.

\section{MESHES AND DISCRETE SPACES}

Let $\mathcal{M}$ be a decomposition of the domain $\Omega$ into quadrangles $(d=2)$ or hexahedra $(d=3)$, supposed to be regular in the usual sense of the finite element literature [4, conditions $\mathcal{T}_{h} 1$ to $\mathcal{T}_{h} 5$, p. 61 and 71]. We denote by $\mathcal{E}$ the set of all faces $\sigma$ of the mesh, by $\mathcal{E}_{\text {ext }}$ the set of faces included in the boundary of $\Omega$, by $\mathcal{E}_{\text {int }}$ the set of internal faces (i.e. $\mathcal{E} \backslash \mathcal{E}_{\text {ext }}$ ) and by $\mathcal{E}(K)$ the faces of a particular cell $K \in \mathcal{M}$. The internal face separating the neighbouring cells $K$ and $L$ is denoted by $\sigma=K \mid L$. Moreover, we also use the notation $\sigma=K \mid$ ext if the face $\sigma$ is included in the boundary of the control volume $K$ and $\sigma$ belongs to $\mathcal{E}_{\text {ext }}$. For each cell $K \in \mathcal{M}$ and each face $\sigma \in \mathcal{E}(K), \boldsymbol{n}_{K, \sigma}$ stands for the unit normal vector to $\sigma$ outward $K$. By $|K|$ and $|\sigma|$, we respectively denote the measures of the control volume $K$ and of the face $\sigma$.

The velocity $\boldsymbol{u}$ and the pressure $p$ are discretized using the so-called Rannacher-Turek element (also called rotated bilinear finite element [27]). The reference element $\hat{K}$ is the unit $d$-cube (with faces orthogonal to the coordinate axes); the discrete functional space on $\hat{K}$ is $\widetilde{\mathbb{Q}_{1}}(\hat{K})$, where $\widetilde{\mathbb{Q}_{1}}(\hat{K})$ is defined as follows:

$$
\widetilde{\mathbb{Q}_{1}}(\hat{K})=\operatorname{span}\left\{1,\left(x_{i}\right)_{i=1, \ldots, d},\left(x_{i}^{2}-x_{i+1}^{2}\right)_{i=1, \cdots, d-1}\right\} .
$$

The mapping $T_{K}$ from the reference element $\hat{K}$ to the current one $K$ is the standard $\mathbb{Q}_{1}$ mapping. Besides, we choose the version of the element where the nodal function $\mathcal{N}_{K, \sigma}$ on the cell $K$ is the average of the velocity through the face $\sigma$ :

$$
\forall K \in \mathcal{M}, \quad \forall \sigma \in \mathcal{E}(K), \quad \forall \boldsymbol{v} \in \mathcal{C}^{\infty}(K), \quad \mathcal{N}_{K, \sigma}(\boldsymbol{v})=\frac{1}{|\sigma|} \int_{\sigma} \boldsymbol{v}_{\mid K} \mathrm{~d} \sigma
$$

Thus the discrete space $X_{h}$ is defined as:

$$
X_{h}=\left\{\boldsymbol{v} \in\left(\mathrm{L}^{2}(\Omega)\right)^{d} ; \forall K \in \mathcal{M}, \boldsymbol{v} \circ T_{K} \in \widetilde{\mathbb{Q}_{1}}(\hat{K})^{d} \text {, and, } \forall \sigma=K \mid L, \mathcal{N}_{K, \sigma}(\boldsymbol{v})=\mathcal{N}_{L, \sigma}(\boldsymbol{v})\right\} .
$$

The approximation for the velocity is thus non-conforming in $\mathrm{H}^{1}(\Omega)^{d}$ because the space $X_{h}$ is composed of discrete functions which are discontinuous through a face, and thus $X_{h} \nsubseteq \mathrm{H}^{1}(\Omega)^{d}$. The set of degrees of freedom for the velocity is:

$$
\left\{\boldsymbol{u}_{\sigma, i}=\mathcal{N}_{\sigma}\left(\boldsymbol{u}_{i}\right), \sigma \in \mathcal{E}, 1 \leq i \leq d\right\},
$$

where $\mathcal{N}_{\sigma}\left(\boldsymbol{u}_{i}\right)=\mathcal{N}_{K, \sigma}\left(\boldsymbol{u}_{i}\right)=\mathcal{N}_{L, \sigma}\left(\boldsymbol{u}_{i}\right)$ if $\sigma=K \mid L$. We denote by $\varphi_{\sigma}^{(i)}$ the vector shape function associated to $\boldsymbol{u}_{\sigma, i}$, which, by definition, reads $\varphi_{\sigma}^{(i)}=\varphi_{\sigma} e^{(i)}$, where $\varphi_{\sigma}$ is the scalar shape function and $\boldsymbol{e}^{(i)}$ is the $i^{\text {th }}$ vector of the canonical basis of $\mathbb{R}^{d}$, and we define $\boldsymbol{u}_{\sigma}$ by $\boldsymbol{u}_{\sigma}=\sum_{i=1}^{d} \boldsymbol{u}_{\sigma, i} \boldsymbol{e}^{(i)}$. With these definitions, we have the identity:

$$
\boldsymbol{u}(\boldsymbol{x})=\sum_{\sigma \in \mathcal{E}} \sum_{i=1}^{d} \boldsymbol{u}_{\sigma, i} \boldsymbol{\varphi}_{\sigma}^{(i)}(\boldsymbol{x})=\sum_{\sigma \in \mathcal{E}} \boldsymbol{u}_{\sigma} \varphi_{\sigma}(\boldsymbol{x}), \quad \text { a.e. in } \Omega .
$$

We respectively denote by $\boldsymbol{\nabla} \boldsymbol{v}$ and $\operatorname{div} \boldsymbol{v}$ the broken gradient and divergence of any function $\boldsymbol{v} \in X_{h}$. 


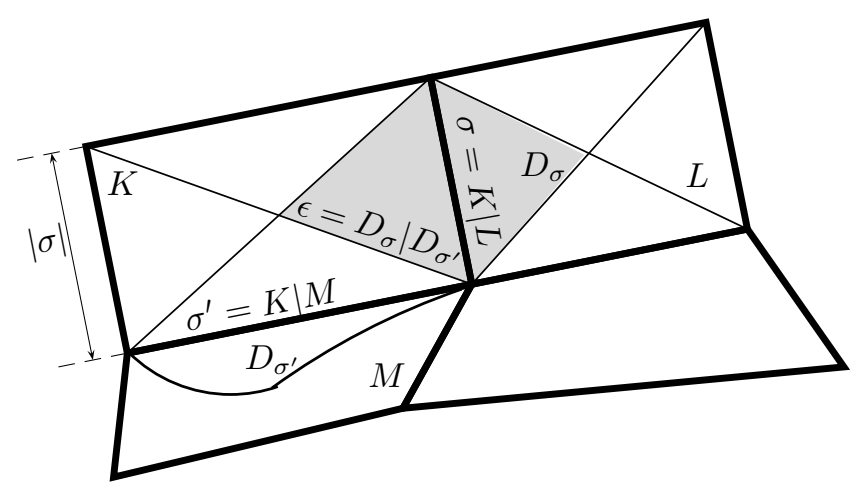

Figure 1. Notations for control volumes and diamond cells.

The pressure belongs to the space $M_{h}$ composed of piecewise constant functions:

$$
M_{h}=\left\{p \in \mathrm{L}^{2}(\Omega) ; \forall K \in \mathcal{M}, p \text { is constant on } K\right\} .
$$

The degrees of freedom for the pressure are consequently $\left\{p_{K} ; K \in \mathcal{M}\right\}$. This pair of finite elements is inf-sup stable [3]. The unknown $\theta$ and the density are also approximated by piecewise constant functions, and their degrees of freedom are denoted by $\left\{\theta_{K} ; K \in \mathcal{M}\right\}$ and $\left\{\rho_{K} ; K \in \mathcal{M}\right\}$.

For the definition of the schemes, we need a dual mesh which is defined as follows. When $K \in \mathcal{M}$ is a rectangle or a cuboid, for $\sigma \in \mathcal{E}(K)$, we define $D_{K, \sigma}$ as the cone with basis $\sigma$ and with vertex the mass center of $K$. We thus obtain a partition of $K$ in $m$ sub-volumes, where $m$ is the number of faces of the cell (i.e. $m=4$ for $d=2$ and $m=6$ for $d=3$ ), each sub-volume having the same measure $\left|D_{K, \sigma}\right|=|K| / m$. We extend this definition to general quadrangles and hexahedra, by supposing that we still have built a partition of equal-volume sub-cells, and with the same connectivities; note that this is of course always possible, but that such a volume $D_{K, \sigma}$ may be no longer a cone, since, if $K$ is far from a parallelogram, it may not be possible to build a cone having $\sigma$ as basis, the opposite vertex lying in $K$ and a volume equal to $|K| / m$. The volume $D_{K, \sigma}$ is referred to as the half-diamond cell associated to $K$ and $\sigma$. For $\sigma \in \mathcal{E}_{\text {int }}, \sigma=K \mid L$, we now define the diamond cell $D_{\sigma}$ associated to $\sigma$ by $D_{\sigma}=D_{K, \sigma} \cup D_{L, \sigma}$ (see Figure 1). For an external face $\sigma \in \mathcal{E}_{\text {ext }} \cap \mathcal{E}(K), D_{\sigma}$ is just the same volume as $D_{K, \sigma}$. We use the following notation for the set of dual faces of the dual mesh thus built: the set of internal dual faces is referred to as $\overline{\mathcal{E}}_{\text {int }}$ and the set of faces of a particular dual cell $D_{\sigma}$ is denoted by $\overline{\mathcal{E}}\left(D_{\sigma}\right)$; the external faces of the dual mesh coincide with primal faces, and the set of dual external faces is thus denoted by $\mathcal{E}_{\text {ext }}$. A dual face $\epsilon$ separating the diamond cells $D_{\sigma}$ and $D_{\sigma^{\prime}}$ is denoted by $\epsilon=D_{\sigma} \mid D_{\sigma^{\prime}}$ and a dual face of $D_{\sigma}$ included in the boundary of the computational domain is denoted by $D_{\sigma} \mid$ ext.

\section{CONSTRUCTION OF A STABLE CONVECTION OPERATOR}

The aim of this section is to establish an analogue of Property K.E. for the velocity convection operator discretized by the Crank-Nicolson time-algorithm.

\subsection{Construction of the operator}

Let us give the main ideas of this construction, details of which are given in [1, 9], by considering the problem at a fixed time step. We start from the discrete mass balance equation, supposed to be obtained by a backward Euler finite volume technique based on the primal mesh (see Section 4 for the description of the whole algorithm):

$$
\forall K \in \mathcal{M}, \quad \frac{|K|}{\delta t}\left(\rho_{K}-\rho_{K}^{*}\right)+\sum_{\sigma \in \mathcal{E}(K)} F_{K, \sigma}=0 .
$$

In this relation, $\rho_{K}$ (resp. $\rho_{K}^{*}$ ) stands for the approximation of the density $\rho$ on the cell $K$ at the end (resp. at the beginning) of the time step, and the quantity $F_{K, \sigma}$ is the discrete mass flux through the 
face $\sigma$ outward $K$, i.e.

$$
F_{K, \sigma}=|\sigma| \tilde{\rho}_{\sigma} \boldsymbol{u}_{\sigma} \cdot \boldsymbol{n}_{K, \sigma},
$$

$\tilde{\rho}_{\sigma}$ being some approximation of the density at the face $\sigma$.

Let $D_{\sigma}$ be a given dual cell. Our aim is to build approximations of the density $\rho_{\sigma}$ and $\rho_{\sigma}^{*}$ and of the mass fluxes through the dual faces of $D_{\sigma},\left(F_{\sigma, \epsilon}\right)_{\epsilon \in \overline{\mathcal{E}}\left(D_{\sigma}\right)}$, such that the following mass balance equation holds:

$$
\frac{\left|D_{\sigma}\right|}{\delta t}\left(\rho_{\sigma}-\rho_{\sigma}^{*}\right)+\sum_{\epsilon \in \overline{\mathcal{E}}\left(D_{\sigma}\right)} F_{\sigma, \epsilon}=0
$$

We first define $\rho_{\sigma}$ by

$$
\begin{cases}\rho_{\sigma}\left|D_{\sigma}\right|=\left|D_{K, \sigma}\right| \rho_{K}+\left|D_{L, \sigma}\right| \rho_{L}, & \text { if } \sigma=K \mid L \in \mathcal{E}_{\text {int }}, \\ \rho_{\sigma}=\rho_{K}, & \text { if } \sigma=K \mid \text { ext } \in \mathcal{E}_{\text {ext }} .\end{cases}
$$

Then, we need to build the fluxes $F_{\sigma, \epsilon}$. The essential idea is to impose the following mass balance over the half dual cell

$$
F_{K, \sigma}+\sum_{\substack{\epsilon \in \overline{\mathcal{E}}\left(D_{\sigma}\right) \\ \epsilon \subset K}} F_{\sigma, \epsilon}=-\frac{\left|D_{K, \sigma}\right|}{\delta t}\left(\rho_{K}-\rho_{K}^{*}\right) .
$$

If we manage to obtain (11) for every $K$ and $\sigma$, then (9) will hold thanks to the following computations:

- For an external face $\sigma$, using that $\rho_{\sigma}=\rho_{K},(11)$ is exactly (9), provided that we choose the primal mass flux $F_{K, \sigma}$ as the dual mass flux through $\sigma$ (remember that $\sigma$ is both a primal and dual face), which we indeed do.

- For an internal face $\sigma=K \mid L$, combining (11) written for $K$ and $L$ with the definition (10) of the density and using the conservativity of the primal mass fluxes (i.e. $F_{K, \sigma}+F_{L, \sigma}=0$ ) yields (9) once again.

Let us now use the primal mass balance (8) to rewrite (11) as

$$
F_{K, \sigma}+\sum_{\substack{\epsilon \in \overline{\mathcal{E}}\left(D_{\sigma}\right) \\ \in \subset K}} F_{\sigma, \epsilon}=\frac{\left|D_{K, \sigma}\right|}{|K|} \sum_{\sigma^{\prime} \in \mathcal{E}(K)} F_{K, \sigma^{\prime}}=\frac{1}{m} \sum_{\sigma^{\prime} \in \mathcal{E}(K)} F_{K, \sigma^{\prime}} .
$$

Writing this relation for all the faces $\sigma$ of $K$, we obtain a linear system for the dual fluxes $\left(F_{\sigma, \epsilon}\right)_{\epsilon \in \overline{\mathcal{E}}\left(D_{\sigma}\right)}$, which is singular (this fact is easily checked by summing these $m$ relations and using the conservativity of the dual mass fluxes). However, thanks to the particular form of the right-hand side of (12), this system has a solution and we can select the one which is orthogonal to the kernel of the system. We obtain an expression of the form

$$
F_{\sigma, \epsilon}=\sum_{\sigma^{\prime} \in \mathcal{E}(K)} \alpha_{\epsilon, \sigma^{\prime}} F_{K, \sigma^{\prime}}
$$

where the coefficients $\left(\alpha_{\epsilon, \sigma^{\prime}}\right)$ are real numbers which appear to be independent of the cell $K$. For instance, in two dimensions and for a cell $K$, if we denote by $F_{\mathrm{N}}, F_{\mathrm{S}}, F_{\mathrm{E}}$ and $F_{\mathrm{W}}$ the four primal mass fluxes and by $F_{\mathrm{W} \mid \mathrm{S}}, F_{\mathrm{S} \mid \mathrm{E}}, F_{\mathrm{E} \mid \mathrm{N}}$ and $F_{\mathrm{N} \mid \mathrm{W}}$ the four dual mass fluxes, as sketched on Figure 2, we obtain expressions of these latter of the form

$$
F_{\sigma, \epsilon}=\alpha_{\mathrm{W}} F_{\mathrm{W}}+\alpha_{\mathrm{E}} F_{\mathrm{E}}+\alpha_{\mathrm{S}} F_{\mathrm{S}}+\alpha_{\mathrm{N}} F_{\mathrm{N}},
$$

with the coefficients gathered in Table I.

We are now in position to define a finite volume convection operator $\mathcal{C}_{h}$ for any discrete fields $\boldsymbol{u}$ and $\boldsymbol{u}^{*}$ in $X_{h}$ as follows:

$$
\forall \sigma \in \mathcal{E}, \quad \mathcal{C}_{h}\left(\boldsymbol{u}, \boldsymbol{u}^{*}\right)_{\sigma}=\frac{1}{\delta t}\left(\rho_{\sigma} \boldsymbol{u}_{\sigma}-\rho_{\sigma}^{*} \boldsymbol{u}_{\sigma}^{*}\right)+\frac{1}{\left|D_{\sigma}\right|} \sum_{\epsilon \in \overline{\mathcal{E}}\left(D_{\sigma}\right)} F_{\sigma, \epsilon} \frac{\boldsymbol{u}_{\epsilon}+\boldsymbol{u}_{\epsilon}^{*}}{2},
$$




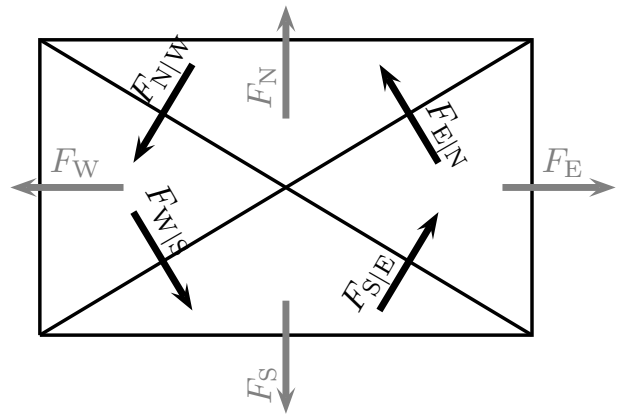

Figure 2. Notation for the mass fluxes.

Table I. Coefficients for the dual mass fluxes in Equation (13).

\begin{tabular}{|l|c|c|c|c|}
\hline$F_{\sigma, \epsilon}$ & $\alpha_{\mathrm{W}}$ & $\alpha_{\mathrm{E}}$ & $\alpha_{\mathrm{S}}$ & $\alpha_{\mathrm{N}}$ \\
\hline$F_{\mathrm{W} \mid \mathrm{S}}$ & $-3 / 8$ & $1 / 8$ & $3 / 8$ & $-1 / 8$ \\
\hline$F_{\mathrm{S} \mid \mathrm{E}}$ & $-1 / 8$ & $3 / 8$ & $-3 / 8$ & $1 / 8$ \\
\hline$F_{\mathrm{E} \mid \mathrm{N}}$ & $1 / 8$ & $-3 / 8$ & $-1 / 8$ & $3 / 8$ \\
\hline$F_{\mathrm{N} \mid \mathrm{W}}$ & $3 / 8$ & $-1 / 8$ & $1 / 8$ & $-3 / 8$ \\
\hline
\end{tabular}

with, for $\epsilon \in \overline{\mathcal{E}}, \boldsymbol{u}_{\epsilon}\left(\right.$ resp. $\left.\boldsymbol{u}_{\epsilon}^{*}\right)$ given as a function of $(\boldsymbol{u})_{\sigma \in \mathcal{E}}\left(\right.$ resp. $\left.\left(\boldsymbol{u}^{*}\right)_{\sigma \in \mathcal{E}}\right)$ by:

$$
\boldsymbol{u}_{\epsilon}= \begin{cases}\frac{1}{2}\left(\boldsymbol{u}_{\sigma}+\boldsymbol{u}_{\sigma^{\prime}}\right), & \text { if } \epsilon=D_{\sigma} \mid D_{\sigma^{\prime}} \in \overline{\mathcal{E}}_{\text {int }}, \\ \boldsymbol{u}_{\sigma}, & \text { if } \epsilon=D_{\sigma} \mid \text { ext } \in \mathcal{E}_{\text {ext }} .\end{cases}
$$

\section{Remark 3.1}

With the same arguments, the construction of the convective operator and the dual mass fluxes can be generalized in three dimensions. For instance, 3D computations are performed in [1].

\subsection{Stability analysis}

In this section, we prove the discrete counterpart of Property K.E. for the convection operator given by (14)-(15).

Proposition 3.2

Let us assume that the discrete mass balance (9) holds, for any $\sigma \in \mathcal{E}$. Provided that $F_{\sigma, \epsilon}=0$ for any $\epsilon \in \mathcal{E}_{\text {ext }}$, we have:

$$
\begin{aligned}
& \forall \sigma \in \mathcal{E}, \quad \forall \boldsymbol{u}, \boldsymbol{u}^{*} \in X_{h}, \\
& \qquad D_{\sigma} \mid \mathcal{C}_{h}\left(\boldsymbol{u}, \boldsymbol{u}^{*}\right)_{\sigma} \cdot\left(\frac{\boldsymbol{u}_{\sigma}+\boldsymbol{u}_{\sigma}^{*}}{2}\right) \\
& \quad=\frac{1}{2 \delta t}\left|D_{\sigma}\right|\left(\rho_{\sigma}\left|\boldsymbol{u}_{\sigma}\right|^{2}-\rho_{\sigma}^{*}\left|\boldsymbol{u}_{\sigma}^{*}\right|^{2}\right)+\frac{1}{2} \sum_{\epsilon=D_{\sigma} \mid D_{\sigma^{\prime}}} F_{\sigma, \epsilon} \frac{\boldsymbol{u}_{\sigma}+\boldsymbol{u}_{\sigma}^{*}}{2} \cdot \frac{\boldsymbol{u}_{\sigma^{\prime}}+\boldsymbol{u}_{\sigma^{\prime}}^{*}}{2}+\mathcal{R}_{\sigma},
\end{aligned}
$$

where $\mathcal{R}_{\sigma}$ is a remainder term given by:

$$
\mathcal{R}_{\sigma}=-\frac{1}{8 \delta t}\left|D_{\sigma}\right|\left(\rho_{\sigma}-\rho_{\sigma}^{*}\right)\left|\boldsymbol{u}_{\sigma}-\boldsymbol{u}_{\sigma}^{*}\right|^{2} .
$$


We recognize at the right-hand side a discrete kinetic energy balance with a conservative finite volume discretization of the kinetic energy convection terms.

Proof

We have:

$$
\left|D_{\sigma}\right| \mathcal{C}_{h}\left(\boldsymbol{u}, \boldsymbol{u}^{*}\right)_{\sigma} \cdot\left(\frac{\boldsymbol{u}_{\sigma}+\boldsymbol{u}_{\sigma}^{*}}{2}\right)=T_{1}+T_{2}
$$

with

$$
\begin{aligned}
& T_{1}=\frac{1}{\delta t}\left|D_{\sigma}\right|\left(\rho_{\sigma} \boldsymbol{u}_{\sigma}-\rho_{\sigma}^{*} \boldsymbol{u}_{\sigma}^{*}\right) \cdot \frac{\boldsymbol{u}_{\sigma}+\boldsymbol{u}_{\sigma}^{*}}{2} \\
& T_{2}=\frac{\boldsymbol{u}_{\sigma}^{*}+\boldsymbol{u}_{\sigma}}{2} \cdot\left(\sum_{\epsilon \in \overline{\mathcal{E}}\left(D_{\sigma}\right)} F_{\sigma, \epsilon} \frac{\boldsymbol{u}_{\epsilon}+\boldsymbol{u}_{\epsilon}^{*}}{2}\right) .
\end{aligned}
$$

The term $T_{1}$ reads:

$$
T_{1}=\underbrace{\frac{1}{2 \delta t}\left|D_{\sigma}\right|\left(\rho_{\sigma}\left|\boldsymbol{u}_{\sigma}\right|^{2}-\rho_{\sigma}^{*}\left|\boldsymbol{u}_{\sigma}^{*}\right|^{2}\right)}_{T_{1,1}}+\underbrace{\frac{1}{2 \delta t}\left|D_{\sigma}\right|\left(\rho_{\sigma}-\rho_{\sigma}^{*}\right) \boldsymbol{u}_{\sigma} \cdot \boldsymbol{u}_{\sigma}^{*}}_{T_{1,2}} .
$$

We now turn to $T_{2}$. Let us introduce the following notation:

$$
\overline{\boldsymbol{u}}_{\sigma}=\frac{\boldsymbol{u}_{\sigma}+\boldsymbol{u}_{\sigma}^{*}}{2}, \forall \sigma \in \mathcal{E} \quad \text { and } \quad \overline{\boldsymbol{u}}_{\epsilon}=\frac{\boldsymbol{u}_{\epsilon}+\boldsymbol{u}_{\epsilon}^{*}}{2}, \forall \epsilon \in \overline{\mathcal{E}}
$$

We get:

$$
T_{2}=\overline{\boldsymbol{u}}_{\sigma} \cdot\left(\sum_{\epsilon \in \overline{\mathcal{E}}\left(D_{\sigma}\right)} F_{\sigma, \epsilon} \overline{\boldsymbol{u}}_{\epsilon}\right) .
$$

Using $\overline{\boldsymbol{u}}_{\sigma} \cdot \overline{\boldsymbol{u}}_{\epsilon}=\left|\overline{\boldsymbol{u}}_{\sigma}\right|^{2}+\overline{\boldsymbol{u}}_{\sigma} \cdot\left(\overline{\boldsymbol{u}}_{\epsilon}-\overline{\boldsymbol{u}}_{\sigma}\right)$, we obtain:

$$
T_{2}=\underbrace{\left|\overline{\boldsymbol{u}}_{\sigma}\right|^{2} \sum_{\epsilon \in \overline{\mathcal{E}}\left(D_{\sigma}\right)} F_{\sigma, \epsilon}}_{T_{2,1}}+\underbrace{\sum_{\epsilon \in \overline{\mathcal{E}}\left(D_{\sigma}\right)} F_{\sigma, \epsilon} \overline{\boldsymbol{u}}_{\sigma} \cdot\left(\overline{\boldsymbol{u}}_{\epsilon}-\overline{\boldsymbol{u}}_{\sigma}\right)}_{T_{2,2}} .
$$

Let us consider the second term $T_{2,2}$. Using the identity $2 \mathbf{a} \cdot(\mathbf{a}-\mathbf{b})=|\mathbf{a}|^{2}+|\mathbf{a}-\mathbf{b}|^{2}-|\mathbf{b}|^{2}$, valid for any real vectors $\mathbf{a}$ and $\mathbf{b}$, we get:

$$
T_{2,2}=-\frac{1}{2}\left|\overline{\boldsymbol{u}}_{\sigma}\right|^{2} \sum_{\epsilon \in \overline{\mathcal{E}}\left(D_{\sigma}\right)} F_{\sigma, \epsilon}+\frac{1}{2} \sum_{\epsilon \in \overline{\mathcal{E}}\left(D_{\sigma}\right)} F_{\sigma, \epsilon}\left(\left|\overline{\boldsymbol{u}}_{\epsilon}\right|^{2}-\left|\overline{\boldsymbol{u}}_{\sigma}-\overline{\boldsymbol{u}}_{\epsilon}\right|^{2}\right) .
$$

Consequently,

$$
T_{2}=\frac{1}{2}\left|\overline{\boldsymbol{u}}_{\sigma}\right|^{2} \sum_{\epsilon \in \overline{\mathcal{E}}\left(D_{\sigma}\right)} F_{\sigma, \epsilon}+\frac{1}{2} \sum_{\epsilon \in \overline{\mathcal{E}}\left(D_{\sigma}\right)} F_{\sigma, \epsilon}\left(\left|\overline{\boldsymbol{u}}_{\epsilon}\right|^{2}-\left|\overline{\boldsymbol{u}}_{\sigma}-\overline{\boldsymbol{u}}_{\epsilon}\right|^{2}\right) .
$$

Using the discrete mass balance equation (9), we get:

$$
T_{2}=-\underbrace{\frac{1}{2 \delta t}\left|D_{\sigma}\right|\left(\rho_{\sigma}-\rho_{\sigma}^{*}\right)\left|\overline{\boldsymbol{u}}_{\sigma}\right|^{2}}_{T_{2,2,1}}+\underbrace{\frac{1}{2} \sum_{\epsilon \in \overline{\mathcal{E}}\left(D_{\sigma}\right)} F_{\sigma, \epsilon}\left(\left|\overline{\boldsymbol{u}}_{\epsilon}\right|^{2}-\left|\overline{\boldsymbol{u}}_{\sigma}-\overline{\boldsymbol{u}}_{\epsilon}\right|^{2}\right)}_{T_{2,2,2}} .
$$

First, the term $T_{2,2,1}$ may be combined with the term $T_{1,2}$ in (16), and using the definition (17) of $\overline{\boldsymbol{u}}_{\sigma}$, this gives:

$$
T_{2,2,1}+T_{1,2}=-\frac{1}{8 \delta t}\left|D_{\sigma}\right|\left(\rho_{\sigma}-\rho_{\sigma}^{*}\right)\left|\boldsymbol{u}_{\sigma}-\boldsymbol{u}_{\sigma}^{*}\right|^{2}=\mathcal{R}_{\sigma} .
$$


Second, by the centered definition (15) of the approximation of the convected velocity at the face, $T_{2,2,2}$ reads also:

$$
T_{2,2,2}=\frac{1}{2} \sum_{\epsilon=D_{\sigma} \mid D_{\sigma^{\prime}}} F_{\sigma, \epsilon} \overline{\boldsymbol{u}}_{\sigma} \cdot \overline{\boldsymbol{u}}_{\sigma^{\prime}}+\frac{1}{2} \sum_{\epsilon=D_{\sigma} \mid \mathrm{ext}} F_{\sigma, \epsilon}\left|\overline{\boldsymbol{u}}_{\sigma}\right|^{2} .
$$

Finally, with Equation (17), we obtain:

$$
T_{2,2,2}=\frac{1}{2} \sum_{\epsilon=D_{\sigma} \mid D_{\sigma^{\prime}}} F_{\sigma, \epsilon} \frac{\left(\boldsymbol{u}_{\sigma}+\boldsymbol{u}_{\sigma}^{*}\right)}{2} \cdot \frac{\left(\boldsymbol{u}_{\sigma^{\prime}}+\boldsymbol{u}_{\sigma^{\prime}}^{*}\right)}{2}+\frac{1}{8}\left|\boldsymbol{u}_{\sigma}+\boldsymbol{u}_{\sigma}^{*}\right|^{2} \sum_{\epsilon=D_{\sigma} \mid \mathrm{ext}} F_{\sigma, \epsilon},
$$

and we conclude the proof invoking the assumption $F_{\sigma, \epsilon}=0$ for any $\epsilon=D_{\sigma} \mid$ ext.

\section{A CRANK-NICOLSON-LIKE PRESSURE CORRECTION SCHEME}

We now present a pressure correction scheme for the resolution of the low Mach number NavierStokes system (1). We begin by presenting the algorithm in the time semi-discrete setting (Section 4.1), then give the space discretization (Section 4.2). Using the property of the convection operator proved in Section 3, we then establish the kinetic energy identity satisfied by the scheme (Section 4.3). In this relation, a remainder term appears when the density is not constant, the time order of which is discussed (Section 4.4).

\subsection{The time-marching algorithm}

We consider a uniform subdivision of the interval $(0, T)$ denoted by $0=t^{0}<t^{1}<\cdots<t^{N}=T$. Let $\delta t$ be the constant time step, namely $\delta t=t^{n+1}-t^{n}$ for any integer $n$ in $\{0, \cdots, N-1\}$.

Let $n$ in $\{0, \cdots, N-1\}$ be fixed and let $p^{n}, \theta^{n}$ and $\rho^{n-1}, \rho^{n}, \boldsymbol{u}^{n}$ be given such that

$$
\frac{\rho^{n}-\rho^{n-1}}{\delta t}+\operatorname{div}\left(\rho^{n} \boldsymbol{u}^{n}\right)=0
$$

The Crank-Nicolson-like time splitting algorithm consists in four steps performed successively:

1- Balance equation for $\theta$ - Solve for $\theta^{n+1}$ :

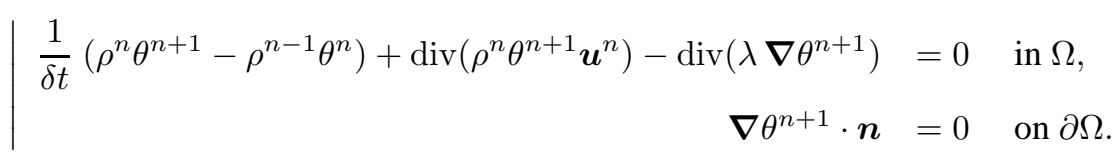

2- Update of the density - Update the density with the equation of state:

$$
\rho^{n+1}=\varrho\left(\theta^{n+1}\right) .
$$

3- Velocity prediction - Solve for $\widetilde{\boldsymbol{u}}^{n+1}$ :

$$
\mid \begin{aligned}
& \frac{1}{\delta t}\left(\rho^{n} \widetilde{\boldsymbol{u}}^{n+1}-\rho^{n-1} \boldsymbol{u}^{n}\right)+\operatorname{div}\left(\widetilde{\boldsymbol{u}}^{n+1 / 2} \otimes \rho^{n} \boldsymbol{u}^{n}\right)-\operatorname{div}\left(\tau\left(\widetilde{\boldsymbol{u}}^{n+1 / 2}\right)\right) \\
&+\boldsymbol{\nabla} p^{n}=0 \text { in } \Omega, \\
& \widetilde{\boldsymbol{u}}^{n+1 / 2} \cdot \boldsymbol{n}=0 \quad \text { and } \quad\left(\tau\left(\widetilde{\boldsymbol{u}}^{n+1 / 2}\right) \cdot \boldsymbol{n}\right) \cdot \boldsymbol{t}=0 \quad \text { on } \partial \Omega,
\end{aligned}
$$

with $\widetilde{\boldsymbol{u}}^{n+1 / 2}=\left(\widetilde{\boldsymbol{u}}^{n+1}+\boldsymbol{u}^{n}\right) / 2$.

4- Velocity and pressure correction - Solve for $\boldsymbol{u}^{n+1}$ and $p^{n+1}$ :

$$
\mid \begin{aligned}
\frac{\rho^{n}}{\delta t}\left(\boldsymbol{u}^{n+1}-\widetilde{\boldsymbol{u}}^{n+1}\right)+\frac{1}{2} \boldsymbol{\nabla}\left(p^{n+1}-p^{n}\right) & =0 & \text { in } \Omega, \\
\frac{1}{\delta t}\left(\rho^{n+1}-\rho^{n}\right)+\operatorname{div}\left(\rho^{n+1} \boldsymbol{u}^{n+1}\right) & =0 & \text { in } \Omega, \\
\boldsymbol{u}^{n+1} \cdot \boldsymbol{n} & =0 & \text { on } \partial \Omega .
\end{aligned}
$$


The first step of the algorithm corresponds to the resolution of the conservation equation for $\theta$ and yields $\theta^{n+1}$; then, thanks to the equation of state, the second step gives the density $\rho^{n+1}$ at time $t^{n+1}=(n+1) \delta t$.

The aim of the third step, called velocity prediction step, is to compute an intermediate velocity field denoted by $\widetilde{\boldsymbol{u}}^{n+1}$, using the pressure $p^{n}$ and the density fields $\rho^{n}$ and $\rho^{n-1}$ already evaluated at the previous time steps. Since we have in view to use the theory developed in Section 3, we need the densities and convection field involved in the convection operator to satisfy a (semi-)discrete mass balance; this latter equation being solved only in Step 4 of the algorithm, we have to rely for this on the mass balance at the previous time step, and we thus are lead to perform a backward time-shift of the densities in the time derivative term.

Finally, the fourth step, referred to as the velocity and pressure correction step, and formulated as a Darcy problem, may seem numerically expensive. In fact, by taking the divergence of the first equation multiplied by $\rho^{n+1} / \rho^{n}$ and using the second one, we are able to eliminate the end-of-step velocity and reformulate this step as an elliptic problem for the pressure increment as follows:

$$
\begin{aligned}
-\frac{\delta t}{2} \operatorname{div}\left(\frac{\rho^{n+1}}{\rho^{n}} \nabla\left(p^{n+1}-p^{n}\right)\right)=-\frac{1}{\delta t}\left(\rho^{n+1}-\rho^{n}\right)-\operatorname{div}\left(\rho^{n+1} \widetilde{\boldsymbol{u}}^{n+1}\right) & \text { in } \Omega, \\
\boldsymbol{\nabla}\left(p^{n+1}-p^{n}\right) \cdot \boldsymbol{n}=0 & \text { on } \partial \Omega .
\end{aligned}
$$

The underlying operator is the Schur complement of the Darcy problem. This computation is actually done at the algebraic level (i.e. equations are first discretized in space before making this computation), which necessitates a priori to compute the inverse of the velocity mass matrix. However, since we adopt here a finite volume discretization of the convection operator (see Section 3 ), the corresponding velocity mass matrix (which is not the usual Rannacher-Turek mass matrix) is diagonal, see (22). Once the pressure increment is known, we deduce the values of the pressure and the end-of-step velocity.

The time accuracy of this algorithm is not analyzed in details here. However, we can see that the system (20) can be understood as a consistent discretization of the following approximate mass balance equation:

$$
\frac{\partial \rho}{\partial t}+\operatorname{div}(\rho \boldsymbol{u})-\frac{\delta t^{2}}{2} \Delta \frac{\partial p}{\partial t}=0
$$

Following the approach proposed in [28] for studying Chorin's projection methods, this remark can certainly lead to a suitable convergence analysis that we may develop in future works. Moreover, we give in Section 5 some numerical evidences that, at least for analytic smooth solutions of the system, our scheme is actually convergent.

Remark 4.1 (Link with a Crank-Nicolson time approximation)

By summing up the velocity prediction (18) and correction (19) equations, we obtain a discrete momentum balance equation with a Crank-Nicolson-like discretization (i.e. with a middle-of-step approximation of the unknowns in the convection, diffusion and pressure gradient terms):

$$
\frac{1}{\delta t}\left(\rho^{n} \boldsymbol{u}^{n+1}-\rho^{n-1} \boldsymbol{u}^{n}\right)+\operatorname{div}\left(\widetilde{\boldsymbol{u}}^{n+1 / 2} \otimes \rho^{n} \boldsymbol{u}^{n}\right)-\operatorname{div}\left(\tau\left(\widetilde{\boldsymbol{u}}^{n+1 / 2}\right)\right)+\nabla p^{n+1 / 2}=0,
$$

with $p^{n+1 / 2}=\left(p^{n+1}+p^{n}\right) / 2$.

Remark 4.2 (Splitting error in time)

This algorithm is formally first order in time when the density varies with time, thus, even if we have derived the prediction and correction steps by using a Crank-Nicolson-like technique, we cannot expect a second order convergence in this case. This would require a more expensive second order splitting technique. Note that our aim here is only to limit the dissipation defect of the scheme.

Remark 4.3 (Convection operator for $\theta$ )

At the continuous level, $\theta$ satisfies a maximum principle. The essential argument which yields this conclusion is that the convection operator in the conservation equation for $\theta$ may be recast, using the mass balance equation, as a transport operator:

$$
\partial_{t}(\rho \theta)+\operatorname{div}(\rho \theta \boldsymbol{u})=\rho\left(\partial_{t} \theta+\boldsymbol{u} \cdot \boldsymbol{\nabla} \theta\right) .
$$


To obtain this property at the discrete level, we thus have to build a convection operator consistent with the mass balance [17] (in other words, which is seen to vanish, invoking the mass balance equation, for constant $\theta$ fields). To this purpose, once again, we have to shift in time the density fields in the time derivative term. Note however that, at the fully discrete level, we fall short to obtain a discrete maximum principle in the general case, since the Laplace operator we use enjoys this property only for specific meshes (see Remark 4.5 below).

\subsection{The fully discrete scheme}

We now give the fully discrete form of the scheme. The construction of the velocity convection operator in the discrete momentum balance equation and the form of the discrete mass balance equation are conform to the developments of Section 3.

Let us assume that $\left(\rho_{K}^{n-1}\right)_{K \in \mathcal{M}},\left(\rho_{K}^{n}\right)_{K \in \mathcal{M}},\left(\theta_{K}^{n}\right)_{K \in \mathcal{M}},\left(\boldsymbol{u}_{\sigma}^{n}\right)_{\sigma \in \mathcal{E}}$ and $\left(p_{K}^{n}\right)_{K \in \mathcal{M}}$ are known, and let us suppose that:

$$
\forall K \in \mathcal{M}, \quad \frac{|K|}{\delta t}\left(\rho_{K}^{n}-\rho_{K}^{n-1}\right)+\sum_{\sigma \in \mathcal{E}(K)} F_{K, \sigma}^{n}=0 .
$$

This relation is the mass balance equation at the previous time step, and the expression of the mass fluxes $\left(F_{K, \sigma}\right)_{\sigma \in \mathcal{E}(K)}$ as a function of the density and the velocity is given below (Step 4 of the algorithm).

Then we perform successively:

1 - Balance equation for $\theta-$ Solve for $\left(\theta_{K}^{n+1}\right)_{K \in \mathcal{M}}$ :

$$
\forall K \in \mathcal{M}, \quad \frac{|K|}{\delta t}\left(\rho_{K}^{n} \theta_{K}^{n+1}-\rho_{K}^{n-1} \theta_{K}^{n}\right)+\sum_{\sigma \in \mathcal{E}(K)} F_{K, \sigma}^{n} \theta_{\sigma}^{n+1}-\left(\operatorname{div}\left(\lambda \nabla \theta^{n+1}\right)\right)_{K}=0,
$$

where $\theta_{\sigma}$ is an upwind approximation for the unknown $\theta$ at the face $\sigma$ and $(\operatorname{div}(\lambda \boldsymbol{\nabla} \cdot))_{K}$ is a finite volume approximation of the (variable coefficient) Laplace operator: for admissible meshes (i.e. here, for structured grids), we use the usual two-point flux approximation, while the variant of the SUSHI method using only cell centered unknowns $[7,26]$ is implemented when the mesh is unstructured.

2- Update of the density - Compute the end-of-step density with the equation of state:

$$
\forall K \in \mathcal{M}, \quad \rho_{K}^{n+1}=\varrho\left(\theta_{K}^{n+1}\right) .
$$

3- Velocity prediction - Disregarding for a while the boundary conditions, this step consists in solving for the predicted velocity unknowns $\left(\widetilde{\boldsymbol{u}}_{\sigma}^{n+1}\right)_{\sigma \in \mathcal{E}}$, the following system:

$$
\begin{aligned}
& \forall \sigma \in \mathcal{E}, \text { for } 1 \leq i \leq d, \\
& \qquad \begin{aligned}
\boldsymbol{e}^{(i)} \cdot\left[\frac{\left|D_{\sigma}\right|}{\delta t}\left(\rho_{\sigma}^{n} \widetilde{\boldsymbol{u}}_{\sigma}^{n+1}-\rho_{\sigma}^{n-1} \boldsymbol{u}_{\sigma}^{n}\right)+\right. & \left.\sum_{\epsilon \in \overline{\mathcal{E}}\left(D_{\sigma}\right)} F_{\sigma, \epsilon}^{n} \widetilde{\boldsymbol{u}}_{\epsilon}^{n+1 / 2}+\left|D_{\sigma}\right|\left(\boldsymbol{\nabla} p^{n}\right)_{\sigma}\right] \\
& +\sum_{K \in \mathcal{M}} \int_{K} \tau\left(\widetilde{\boldsymbol{u}}^{n+1 / 2}\right): \nabla \boldsymbol{\varphi}_{\sigma}^{(i)} \mathrm{d} \boldsymbol{x}=0,
\end{aligned}
\end{aligned}
$$

where we recall that $\widetilde{\boldsymbol{u}}^{n+1 / 2}=\left(\widetilde{\boldsymbol{u}}^{n+1}+\boldsymbol{u}^{n}\right) / 2$. The convection operator (i.e. the computation of the density at the faces $\rho_{\sigma}$, of the mass fluxes at the dual faces $\left(F_{\sigma, \epsilon}\right)$ and the (centered) approximation $\widetilde{\boldsymbol{u}}_{\epsilon}^{n+1 / 2}$ of the velocity at the dual faces) is described in Section 3.1. The pressure gradient term reads:

$$
\left|D_{\sigma}\right|\left(\boldsymbol{\nabla} p^{n}\right)_{\sigma} \cdot \boldsymbol{e}^{(i)}=-\sum_{K \in \mathcal{M}} \int_{K} p^{n} \operatorname{div} \boldsymbol{\varphi}_{\sigma}^{(i)} \mathrm{d} \boldsymbol{x},
$$


and thus, for internal faces,

$$
\left|D_{\sigma}\right|\left(\nabla p^{n}\right)_{\sigma}=|\sigma|\left(p_{L}^{n}-p_{K}^{n}\right) \boldsymbol{n}_{K, \sigma}, \quad \forall \sigma \in \mathcal{E}_{\text {int }}, \sigma=K \mid L .
$$

On an external face, the impermeability boundary condition must be taken into account by (possibly, i.e. if the normal of the face is not collinear to a coordinate axis) making a change of unknowns for the velocity to make appear the component(s) of the velocity tangent and normal to the boundary, and prescribing this latter to zero; at each $\sigma \in \mathcal{E}_{\text {ext }}$, an equation is thus suppressed from the system. Since the pressure gradient at a face $\sigma$ is normal to $\sigma$, it is thus useless to define it for $\sigma \in \mathcal{E}_{\text {ext }}$. Note that, since the Neumann boundary condition associated to the tangential component(s) is homogeneous, no extra surface term appears from the integration by parts of the viscous term.

4- Velocity and pressure correction step - Solve for the end-of-step velocity unknowns $\left(\boldsymbol{u}_{\sigma}^{n+1}\right)_{\sigma \in \mathcal{E}}$ and the pressure unknowns $\left(p_{K}^{n+1}\right)_{K \in \mathcal{M}}$ the following system:

$$
\mid \begin{array}{ll}
\forall \sigma \in \mathcal{E}_{\text {int }}, & \frac{\left|D_{\sigma}\right|}{\delta t} \rho_{\sigma}^{n}\left(\boldsymbol{u}_{\sigma}^{n+1}-\widetilde{\boldsymbol{u}}_{\sigma}^{n+1}\right)+\frac{1}{2}\left|D_{\sigma}\right|\left(\nabla\left(p^{n+1}-p^{n}\right)\right)_{\sigma}=0, \\
\forall K \in \mathcal{M}, & \frac{|K|}{\delta t}\left(\rho_{K}^{n+1}-\rho_{K}^{n}\right)+\sum_{\sigma \in \mathcal{E}(K)} F_{K, \sigma}^{n+1}=0 .
\end{array}
$$

Note that, since the pressure gradient is normal to a face and the velocity normal to the boundary is prescribed to zero, the velocity on the boundary is left unchanged, at this step. This explains why the system associated to the velocity correction (first equation of (22)) may be restricted to internal faces.

Since the density is given by the equation of state, there is no need to use upwinding in the mass balance equation (by opposition with compressible flows problem [14], for instance, where upwinding ensures the positivity of $\rho$ ), and we thus use a centered approach:

$$
\begin{array}{ll}
\forall \sigma \in \mathcal{E}_{\text {int }}, \sigma=K \mid L, & F_{K, \sigma}^{n+1}=|\sigma| \frac{\left(\rho_{K}^{n+1}+\rho_{L}^{n+1}\right)}{2} \boldsymbol{u}_{\sigma}^{n+1} \cdot \boldsymbol{n}_{K, \sigma}, \\
\forall \sigma \in \mathcal{E}_{\text {ext }}, \sigma=K \mid \text { ext }, & F_{K, \sigma}^{n+1}=0 .
\end{array}
$$

Remark 4.4 (Spatial order of accuracy)

Despite the fact that we use a finite volume approximation of the convective operator instead of its finite element form, the overall accuracy of the method is preserved, see Section 5.1.

Remark 4.5 (Maximum principle for $\theta$ and positivity of the density)

For structured meshes, the upwind choice for $\theta$ in the discrete convection operator of the associated balance conservation equation, together with the particular form of the Laplace operator, ensure a discrete maximum principle, and thus the positivity of the density. When the mesh is unstructured, no such property is known for the SUSHI scheme. However, we did not observe under- or overshoots in practical computations.

\subsection{The discrete kinetic energy identity}

We first define the following density-dependent discrete semi-norm for the pressure $p$ in $M_{h}$ :

$$
|p|_{1, \mathcal{M}, \rho}^{2}=\sum_{\sigma \in \mathcal{E}_{\mathrm{int}}} \frac{1}{\rho_{\sigma}\left|D_{\sigma}\right|}\left|(\nabla p)_{\sigma}\right|^{2}=\sum_{\substack{\sigma \in \mathcal{E}_{\text {int }}, \sigma=K \mid L}} \frac{|\sigma|^{2}}{\rho_{\sigma}\left|D_{\sigma}\right|}\left(p_{K}-p_{L}\right)^{2} .
$$

This semi-norm is well defined as soon as the density is positive, which is always the case in practice (see Remark 4.5).

The application of the discrete counterpart of the continuous procedure described in introduction yields the following result. 
Proposition 4.6 (Global kinetic energy balance)

Let us assume that $F_{\sigma, \epsilon}=0$ for any $\epsilon \in \mathcal{E}_{\text {ext }}$. Provided that the discrete mass balance (9) holds, we have, for $0 \leq n \leq N-1$ :

$$
\begin{aligned}
& \frac{1}{2} \sum_{\sigma \in \mathcal{E}}\left|D_{\sigma}\right|\left(\rho_{\sigma}^{n}\left|\boldsymbol{u}_{\sigma}^{n+1}\right|^{2}-\rho_{\sigma}^{n-1}\left|\boldsymbol{u}_{\sigma}^{n}\right|^{2}\right)+\delta t \sum_{K \in \mathcal{M}} \int_{K} \tau\left(\widetilde{\boldsymbol{u}}^{n+1 / 2}\right): \nabla \widetilde{\boldsymbol{u}}^{n+1 / 2} \mathrm{~d} \boldsymbol{x} \\
& \quad-\frac{\delta t}{2} \sum_{K \in \mathcal{M}}\left[p_{K}^{n}\left(\sum_{\sigma \in \mathcal{E}(K)}|\sigma| \boldsymbol{u}_{\sigma}^{n} \cdot \boldsymbol{n}_{K, \sigma}\right)+p_{K}^{n+1}\left(\sum_{\sigma \in \mathcal{E}(K)}|\sigma| \boldsymbol{u}_{\sigma}^{n+1} \cdot \boldsymbol{n}_{K, \sigma}\right)\right]+\mathcal{D}^{n+1}=0,
\end{aligned}
$$

where $\mathcal{D}^{n+1}$ stands for a numerical remainder term which reads

$$
\mathcal{D}^{n+1}=\frac{\delta t^{2}}{8}\left(\left|p^{n+1}\right|_{1, \mathcal{M}, \rho^{n}}^{2}-\left|p^{n}\right|_{1, \mathcal{M}, \rho^{n}}^{2}\right)-\frac{1}{8} \sum_{\sigma \in \mathcal{E}}\left|D_{\sigma}\right|\left(\rho_{\sigma}^{n}-\rho_{\sigma}^{n-1}\right)\left|\widetilde{\boldsymbol{u}}_{\sigma}^{n+1}-\boldsymbol{u}_{\sigma}^{n}\right|^{2} .
$$

Proof

Let $n \in\{0, \ldots, N-1\}$ and $\sigma \in \mathcal{E}$. Let us multiply the discrete velocity prediction step (21) associated to the face $\sigma$ and the component $i$ by $\left(\widetilde{\boldsymbol{u}}_{\sigma}^{n+1 / 2}\right)_{i}$ for $1 \leq i \leq d$ and then sum over $i$. Invoking Proposition 3.2, we get

$$
\begin{aligned}
& \frac{\left|D_{\sigma}\right|}{2 \delta t}\left(\rho_{\sigma}^{n}\left|\widetilde{\boldsymbol{u}}_{\sigma}^{n+1}\right|^{2}-\rho_{\sigma}^{n-1}\left|\boldsymbol{u}_{\sigma}^{n}\right|^{2}\right)+\frac{1}{2} \sum_{\epsilon=D_{\sigma} \mid D_{\sigma^{\prime}}} F_{\sigma, \epsilon}^{n} \widetilde{\boldsymbol{u}}_{\sigma}^{n+1 / 2} \cdot \widetilde{\boldsymbol{u}}_{\sigma^{\prime}}^{n+1 / 2} \\
& \quad+\left|D_{\sigma}\right|\left(\boldsymbol{\nabla} p^{n}\right)_{\sigma} \cdot \widetilde{\boldsymbol{u}}_{\sigma}^{n+1 / 2}+\left|D_{\sigma}\right|\left(-\operatorname{div} \tau\left(\widetilde{\boldsymbol{u}}^{n+1 / 2}\right)\right)_{\sigma} \cdot \widetilde{\boldsymbol{u}}_{\sigma}^{n+1 / 2}+\mathcal{R}_{\sigma}^{n+1}=0,
\end{aligned}
$$

where the discrete approximation of the viscous diffusion operator applied to $\widetilde{\boldsymbol{u}}^{n+1 / 2}$ reads

$$
\text { for } 1 \leq i \leq d, \text { for } \sigma \in \mathcal{E}, \quad\left|D_{\sigma}\right|\left(-\operatorname{div} \tau\left(\widetilde{\boldsymbol{u}}^{n+1 / 2}\right)\right)_{\sigma, i}=\sum_{K \in \mathcal{M}} \int_{K} \tau\left(\widetilde{\boldsymbol{u}}^{n+1 / 2}\right): \nabla \boldsymbol{\varphi}_{\sigma}^{(i)} \mathrm{d} \boldsymbol{x},
$$

and the remainder term $\mathcal{R}_{\sigma}^{n+1}$ reads

$$
\mathcal{R}_{\sigma}^{n+1}=-\frac{\left|D_{\sigma}\right|}{8 \delta t}\left(\rho_{\sigma}^{n}-\rho_{\sigma}^{n-1}\right)\left|\widetilde{\boldsymbol{u}}_{\sigma}^{n+1}-\boldsymbol{u}_{\sigma}^{n}\right|^{2} .
$$

We now recast the first equation of the correction step (22) as follows:

$$
\left[\frac{\left|D_{\sigma}\right| \rho_{\sigma}^{n}}{2 \delta t}\right]^{1 / 2} \boldsymbol{u}_{\sigma}^{n+1}+\left[\frac{\left|D_{\sigma}\right| \delta t}{8 \rho_{\sigma}^{n}}\right]^{1 / 2}\left(\nabla p^{n+1}\right)_{\sigma}=\left[\frac{\left|D_{\sigma}\right| \rho_{\sigma}^{n}}{2 \delta t}\right]^{1 / 2} \widetilde{\boldsymbol{u}}_{\sigma}^{n+1}+\left[\frac{\left|D_{\sigma}\right| \delta t}{8 \rho_{\sigma}^{n}}\right]^{1 / 2}\left(\boldsymbol{\nabla} p^{n}\right)_{\sigma} .
$$

Taking the square of the norm of both sides of this relation, we get

$$
\begin{aligned}
\frac{\left|D_{\sigma}\right|}{2 \delta t} \rho_{\sigma}^{n}\left|\boldsymbol{u}_{\sigma}^{n+1}\right|^{2}+\frac{1}{2}\left|D_{\sigma}\right| & \left(\boldsymbol{\nabla} p^{n+1}\right)_{\sigma} \cdot \boldsymbol{u}_{\sigma}^{n+1}+\frac{\delta t}{8} \frac{\left|D_{\sigma}\right|}{\rho_{\sigma}^{n}}\left|\left(\boldsymbol{\nabla} p^{n+1}\right)_{\sigma}\right|^{2} \\
= & \frac{\left|D_{\sigma}\right|}{2 \delta t} \rho_{\sigma}^{n}\left|\widetilde{\boldsymbol{u}}_{\sigma}^{n+1}\right|^{2}+\frac{1}{2}\left|D_{\sigma}\right|\left(\boldsymbol{\nabla} p^{n}\right)_{\sigma} \cdot \widetilde{\boldsymbol{u}}_{\sigma}^{n+1}+\frac{\delta t}{8} \frac{\left|D_{\sigma}\right|}{\rho_{\sigma}^{n}}\left|\left(\boldsymbol{\nabla} p^{n}\right)_{\sigma}\right|^{2} .
\end{aligned}
$$

Summing with (26) and multiplying by $\delta t$, we obtain

$$
\begin{aligned}
\frac{\left|D_{\sigma}\right|}{2}\left(\rho_{\sigma}^{n}\left|\boldsymbol{u}_{\sigma}^{n+1}\right|^{2}-\rho_{\sigma}^{n-1}\left|\boldsymbol{u}_{\sigma}^{n}\right|^{2}\right)+ & \frac{\delta t}{2} \sum_{\epsilon=D_{\sigma} \mid D_{\sigma^{\prime}}} F_{\sigma, \epsilon}^{n} \widetilde{\boldsymbol{u}}_{\sigma}^{n+1 / 2} \cdot \widetilde{\boldsymbol{u}}_{\sigma^{\prime}}^{n+1 / 2} \\
+\frac{\delta t\left|D_{\sigma}\right|}{2}[ & \left.\left(\boldsymbol{\nabla} p^{n}\right)_{\sigma} \cdot \boldsymbol{u}_{\sigma}^{n}+\left(\boldsymbol{\nabla} p^{n+1}\right)_{\sigma} \cdot \boldsymbol{u}_{\sigma}^{n+1}\right] \\
& \quad+\left|D_{\sigma}\right| \delta t\left(-\operatorname{div} \tau\left(\widetilde{\boldsymbol{u}}^{n+1 / 2}\right)\right)_{\sigma} \cdot \widetilde{\boldsymbol{u}}_{\sigma}^{n+1 / 2}+\mathcal{D}_{\sigma}^{n+1}=0,
\end{aligned}
$$


with

$$
\mathcal{D}_{\sigma}^{n+1}=-\frac{\left|D_{\sigma}\right|}{8}\left(\rho_{\sigma}^{n}-\rho_{\sigma}^{n-1}\right)\left|\widetilde{\boldsymbol{u}}_{\sigma}^{n+1}-\boldsymbol{u}_{\sigma}^{n}\right|^{2}+\frac{\delta t^{2}}{8}\left(\frac{\left|D_{\sigma}\right|}{\rho_{\sigma}^{n}}\left|\left(\boldsymbol{\nabla} p^{n+1}\right)_{\sigma}\right|^{2}-\frac{\left|D_{\sigma}\right|}{\rho_{\sigma}^{n}}\left|\left(\boldsymbol{\nabla} p^{n}\right)_{\sigma}\right|^{2}\right) .
$$

We then sum over all the faces. The kinetic energy convection term (i.e. the second term at the left-hand side) vanishes by conservativity (for $\epsilon=D_{\sigma} \mid D_{\sigma^{\prime}}, F_{\sigma, \epsilon}^{n}=-F_{\sigma^{\prime}, \epsilon}^{n}$ ) and by the boundary conditions (for $\epsilon=D_{\sigma} \mid$ ext, $F_{\sigma, \epsilon}^{n}=0$ ). We make a discrete integration by parts in the gradient terms, i.e. we use the fact that, thanks to the definition of the pressure gradient and, once again, to the boundary conditions, for any discrete functions $\boldsymbol{u}$ and $p$,

$$
\sum_{\substack{\sigma \in \mathcal{E}_{\text {int }}, \sigma=K \mid L}}\left|D_{\sigma}\right|(\boldsymbol{\nabla} p)_{\sigma} \cdot \boldsymbol{u}_{\sigma}=\sum_{\substack{\sigma \in \mathcal{E}_{\text {int }}, \sigma=K \mid L}}|\sigma|\left(p_{L}-p_{K}\right) \boldsymbol{n}_{K, \sigma} \cdot \boldsymbol{u}_{\sigma}=-\sum_{K \in \mathcal{M}} p_{K} \sum_{\sigma \in \mathcal{E}(K)}|\sigma| \boldsymbol{u}_{\sigma} \cdot \boldsymbol{n}_{K, \sigma} .
$$

Finally, we easily get that, by definition,

$$
\sum_{\sigma \in \mathcal{E}}\left|D_{\sigma}\right|\left(-\operatorname{div} \tau\left(\widetilde{\boldsymbol{u}}^{n+1 / 2}\right)\right)_{\sigma} \cdot \widetilde{\boldsymbol{u}}_{\sigma}^{n+1 / 2}=\sum_{K \in \mathcal{M}} \int_{K} \tau\left(\widetilde{\boldsymbol{u}}^{n+1 / 2}\right): \nabla \widetilde{\boldsymbol{u}}^{n+1 / 2} \mathrm{~d} \boldsymbol{x},
$$

which concludes the proof.

Remark 4.7 (Local kinetic energy identity)

Note that Equation (27) is the discrete analogue of the (local) kinetic energy identity (6), to which it may be identified term by term. Both the kinetic energy flux (second term) and the pressure gradient term (third one) are consistent with centered in time and space discretizations.

Such a relation may be used for different purposes. For instance, one may find in [14] a way to exploit such an identity to switch in Euler equations from the total energy to the internal energy balance without loosing the consistency of the scheme.

\subsection{Order in time of the dissipation defect}

In the previous subsection, it has been shown (Proposition 4.6) that we are able to write a discrete equation governing the variation of kinetic energy for the Crank-Nicolson-like pressure correction scheme presented before. Adding up this relation (i.e. Equation (24)) for $n$ ranging from 0 to $N-1$, we get

$$
\begin{aligned}
\frac{1}{2} \sum_{\sigma \in \mathcal{E}}\left|D_{\sigma}\right|\left(\rho_{\sigma}^{N-1}\left|\boldsymbol{u}_{\sigma}^{N}\right|^{2}-\rho_{\sigma}^{-1}\left|\boldsymbol{u}_{\sigma}^{0}\right|^{2}\right)+\delta t \sum_{n=0}^{N-1} \sum_{K \in \mathcal{M}} \int_{K} \tau\left(\widetilde{\boldsymbol{u}}^{n+1 / 2}\right): \nabla \widetilde{\boldsymbol{u}}^{n+1 / 2} \mathrm{~d} \boldsymbol{x} \\
-\frac{\delta t}{2} \sum_{n=0}^{N-1} \sum_{K \in \mathcal{M}} \sum_{\sigma \in \mathcal{E}(K)}|\sigma|\left(p_{K}^{n} \boldsymbol{u}_{\sigma}^{n}+p_{K}^{n+1} \boldsymbol{u}_{\sigma}^{n+1}\right) \cdot \boldsymbol{n}_{K, \sigma}+\mathcal{D}=0,
\end{aligned}
$$

with

$$
\mathcal{D}=\frac{\delta t^{2}}{8} \sum_{n=0}^{N-1}\left(\left|p^{n+1}\right|_{1, \mathcal{M}, \rho^{n}}^{2}-\left|p^{n}\right|_{1, \mathcal{M}, \rho^{n}}^{2}\right)-\frac{1}{8} \sum_{n=0}^{N-1} \sum_{\sigma \in \mathcal{E}}\left|D_{\sigma}\right|\left(\rho_{\sigma}^{n}-\rho_{\sigma}^{n-1}\right)\left|\widetilde{\boldsymbol{u}}_{\sigma}^{n+1}-\boldsymbol{u}_{\sigma}^{n}\right|^{2} .
$$

Relation (28) does not provide a stability estimate, for three reasons

1 - The third term at the left-hand side is the discrete counterpart of the continuous term $\int_{0}^{T} \int_{\Omega} p \operatorname{div} \boldsymbol{u} \mathrm{d} \boldsymbol{x} \mathrm{d} t$, the control of which is not possible in the low Mach number model; for an incompressible flow, this term would simply vanish.

2 - The first term in the remainder $\mathcal{D}$ is not non-negative, because of the presence of different density weights in pressure semi-norms; this problem would be cured if the quantity $\left|p^{n}\right|_{1, \mathcal{M}, \rho^{n}}$ was replaced by $\left|p^{n}\right|_{1, \mathcal{M}, \rho^{n-1}}$, and this can be done by adding to the algorithm a pressure renormalization step, following the study presented in [11]. This step is not implemented here, because it does not seem to be crucial for the robustness of our scheme. 
3 - The last term in $\mathcal{D}$ can take any sign.

Note that none of these issues occurs when the density is constant $(\operatorname{div} \boldsymbol{u}=0$ and $\mathcal{D}=0)$, so that the scheme is unconditionally stable in this case.

The quantity $\mathcal{D}$ is a numerical remainder term, which is referred to hereafter as the dissipation defect of the scheme. We now show that $\mathcal{D}$ is formally second order in time. Indeed, we have, using Equation (23) and reordering the sum in time

$$
\begin{aligned}
\frac{\delta t^{2}}{8} \sum_{n=0}^{N-1}\left(\left|p^{n+1}\right|_{1, \mathcal{M}, \rho^{n}}^{2}-\left|p^{n}\right|_{1, \mathcal{M}, \rho^{n}}^{2}\right)= & \frac{\delta t^{2}}{8}\left(\left|p^{N}\right|_{1, \mathcal{M}, \rho^{N-1}}^{2}\left|p^{0}\right|_{1, \mathcal{M}, \rho^{0}}^{2}\right) \\
& +\frac{\delta t^{2}}{8} \sum_{n=1}^{N-1} \sum_{\sigma \in \mathcal{E}_{\text {int }}}\left|D_{\sigma}\right|\left|\left(\nabla p^{n}\right)_{\sigma}\right|^{2}\left(\frac{1}{\rho_{\sigma}^{n-1}}-\frac{1}{\rho_{\sigma}^{n}}\right) .
\end{aligned}
$$

Therefore, each sum in $\mathcal{D}$ is a product of $\delta t^{2}$ multiplied by a sum that behaves like $\mathcal{O}(1)$ in time.

Remark 4.8 (First order backward Euler time discretization)

When approximating the velocity time derivative with first order backward Euler scheme [1], a similar analysis yields, for $0 \leq n \leq N-1$

$$
\begin{aligned}
\frac{1}{2} \sum_{\sigma \in \mathcal{E}}\left|D_{\sigma}\right|\left(\rho_{\sigma}^{n}\left|\boldsymbol{u}_{\sigma}^{n+1}\right|^{2}-\rho_{\sigma}^{n-1}\left|\boldsymbol{u}_{\sigma}^{n}\right|^{2}\right) & +\delta t \sum_{K \in \mathcal{M}} \int_{K} \tau\left(\widetilde{\boldsymbol{u}}^{n+1}\right): \nabla \widetilde{\boldsymbol{u}}^{n+1} \mathrm{~d} \boldsymbol{x} \\
& -\delta t \sum_{K \in \mathcal{M}} p_{K}^{n+1} \sum_{\sigma \in \mathcal{E}(K)}|\sigma| \boldsymbol{u}_{\sigma}^{n+1} \cdot \boldsymbol{n}_{K, \sigma}+\mathcal{D}_{\text {Euler }}^{n+1}=0,
\end{aligned}
$$

where the numerical remainder term $\mathcal{D}_{\text {Euler }}^{n+1}$ now reads

$$
\mathcal{D}_{\text {Euler }}^{n+1}=\frac{\delta t^{2}}{2}\left(\left|p^{n+1}\right|_{1, \mathcal{M}, \rho^{n}}^{2}-\left|p^{n}\right|_{1, \mathcal{M}, \rho^{n}}^{2}\right)+\frac{1}{2} \sum_{\sigma \in \mathcal{E}}\left|D_{\sigma}\right| \rho_{\sigma}^{n}\left|\widetilde{\boldsymbol{u}}_{\sigma}^{n+1}-\boldsymbol{u}_{\sigma}^{n}\right|^{2} .
$$

Summing up Equalities (29) for $n$ ranging from 0 to $N-1$, we get the following expression for the dissipation defect of the scheme

$$
\mathcal{D}_{\text {Euler }}=\frac{\delta t^{2}}{2} \sum_{n=0}^{N-1}\left(\left|p^{n+1}\right|_{1, \mathcal{M}, \rho^{n}}^{2}-\left|p^{n}\right|_{1, \mathcal{M}, \rho^{n}}^{2}\right)+\frac{1}{2} \sum_{n=0}^{N-1} \sum_{\sigma \in \mathcal{E}}\left|D_{\sigma}\right| \rho_{\sigma}^{n}\left|\widetilde{\boldsymbol{u}}_{\sigma}^{n+1}-\boldsymbol{u}_{\sigma}^{n}\right|^{2},
$$

and, if, as in the Crank-Nicolson-like pressure correction scheme, the first sum behaves like $\mathcal{O}\left(\delta t^{2}\right)$, the second one behaves as $\mathcal{O}(\delta t)$ and the dissipation defect is thus first order in time.

\section{NUMERICAL EXPERIMENTS}

In this section, we report some numerical experiments performed to assess the behaviour of the Crank-Nicolson-like pressure correction scheme proposed in this paper. To this purpose, we compare it to a classical pressure correction scheme [1], based on the same space discretization and on the first order backward Euler approximation in time. The simulations are performed with the ISIS software [15] based on the development platform and software component library PELICANS [25], both developed at IRSN (Institut de Radioprotection et de Sûreté Nucléaire).

\subsection{Convergence and dissipation defect}

We address here a test case with an analytical solution, in order to assess the convergence properties of the scheme under study. We also check that the behaviour of the dissipation defect with respect to the time step is consistent with the theoretical results of Section 4.4, for both Euler and CrankNicolson time discretizations. 
We consider the system (1)-(2), with the following expression for the density $\rho$ as a function of the unknown $\theta$ :

$$
\rho=\varrho(\theta)=\frac{1}{\frac{\theta}{\rho_{1}}+\frac{1-\theta}{\rho_{2}}} .
$$

With this closure laws, the system at hand may be seen as the set of equations governing a twocomponent flow, of component densities $\rho_{1}$ and $\rho_{2}$ (here supposed to be two constant positive real numbers); the unknown $\theta$ stands for the mass fraction of the first component.

Let us suppose that the normal velocity is prescribed to zero on the whole boundary of the computational domain $\partial \Omega$. In this condition, integrating the mass balance over $\Omega$ yields the total mass balance:

$$
\frac{d}{d t} \int_{\Omega} \rho \mathrm{d} \boldsymbol{x}=0
$$

On the other side, the density $\rho$ is given as a function of $\theta$, itself solution of Equation (2) for $\lambda=10^{-4}$. Integrating this relation over $\Omega$ and supposing that the mass diffusion flux of the first component vanishes at the boundary, i.e. $\lambda \boldsymbol{\nabla} \theta \cdot \boldsymbol{n}=0$ on $\partial \Omega$, we obtain:

$$
\frac{d}{d t} \int_{\Omega} \rho \theta \mathrm{d} \boldsymbol{x}=0
$$

By an easy manipulation of the equation of state (31), we get:

$$
\rho=\rho_{2}+\left(1-\frac{\rho_{2}}{\rho_{1}}\right) \rho \theta
$$

and thus, since $\rho$ appears as an affine function of $\rho \theta$, the relations (32) and (33) are fortunately compatible. With the proposed time-stepping algorithm, this property does not hold anymore, because of the time shift of the density in the solution of the balance equation for $\theta$. Consequently, a renormalisation of the density is necessary to ensure the existence of a solution to the projection step:

$$
\rho^{n+1}(\boldsymbol{x})=\left[\frac{\int_{\Omega} \rho^{n} \mathrm{~d} \boldsymbol{x}}{\int_{\Omega} \varrho\left(\theta^{n+1}\right) \mathrm{d} \boldsymbol{x}}\right] \varrho\left(\theta^{n+1}(\boldsymbol{x})\right) .
$$

Note that this relation is reminiscent of the scaling of the density obtained through its dependency versus the so-called thermodynamical pressure in the asymptotic model for low Mach number flows [19].

We consider now the following solution to System (1)-(2):

$$
\begin{aligned}
& \rho(\boldsymbol{x}, t)=1+\frac{1}{4} \cos (\pi t)\left[\sin \left(\pi \boldsymbol{x}_{1}\right)+\sin \left(\pi \boldsymbol{x}_{2}\right)\right], \\
& \rho(\boldsymbol{x}, t) \boldsymbol{u}(\boldsymbol{x}, t)=-\frac{1}{4} \sin (\pi t)\left[\begin{array}{l}
\cos \left(\pi \boldsymbol{x}_{1}\right) \\
\cos \left(\pi \boldsymbol{x}_{2}\right)
\end{array}\right] \\
& p(\boldsymbol{x}, t)=\cos (\pi t)\left[\cos \left(\pi \boldsymbol{x}_{1}\right)+\cos \left(\pi \boldsymbol{x}_{2}\right)\right] .
\end{aligned}
$$

We suppose that $\rho_{1}=0.25$ and $\rho_{2}=2$, so relation (31) yields $\theta=(2-\rho) /(7 \rho)$. The viscosity is supposed to be $\mu=0.001$. The computational domain is $\Omega=(0,1) \times(0,1)$, so the normal velocity is always zero at the boundary, and the integral of the density over $\Omega$ does not vary with time. With this choice for $\rho$ and $\rho \boldsymbol{u}$, the mass balance is satisfied. The right-hand side in the momentum balance and in the $\theta$ transport equation, the initial conditions and the boundary conditions (prescribed value for $\boldsymbol{u}$ ) are chosen to match the analytical solution. The non zero right-hand side for this system is numerically treated implicitly.

The domain is meshed by $n \times n$ regular grids, with $n=16,32,64$ and 128 . The discretization of the convection term for $\theta$ is performed by an upwind finite volume scheme. Errors obtained at $t=0.5$ are displayed on figures 3,4 and 5 , in the $\mathrm{L}^{2}$ norm for the velocity and the pressure, and, for 


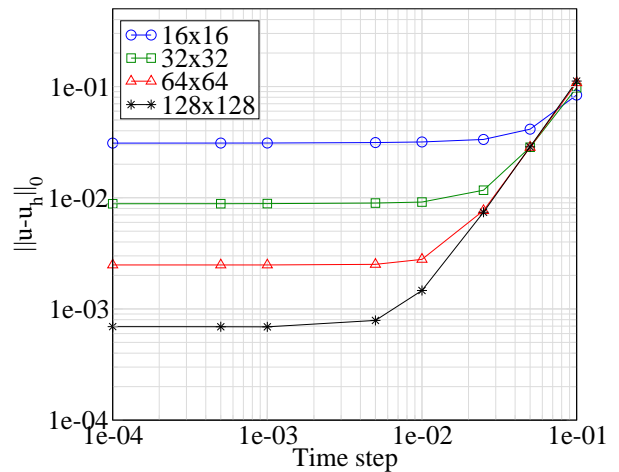

(a) Euler.

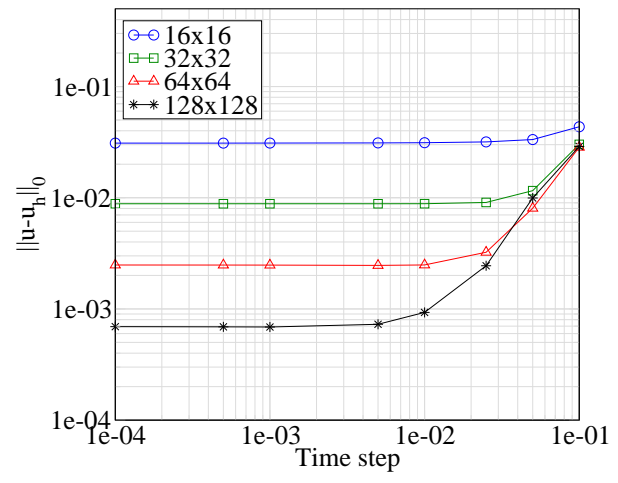

(b) Crank-Nicolson-like.

Figure 3. Test with a known solution: time convergence in $\mathrm{L}^{2}$ norm for the velocity, for various meshes.

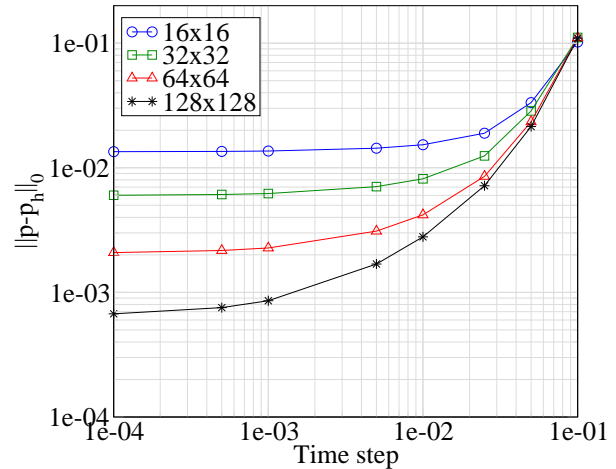

(a) Euler.

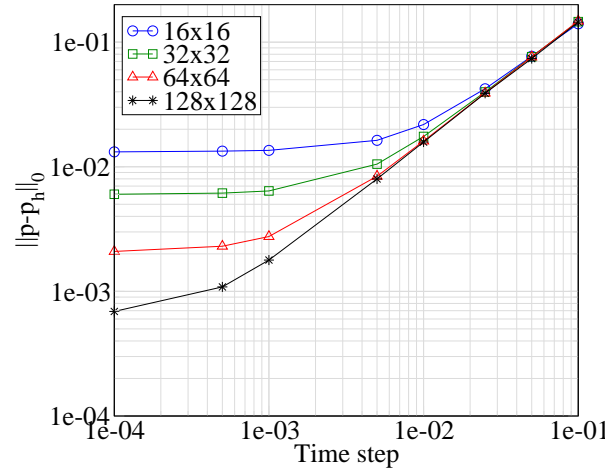

(b) Crank-Nicolson-like.

Figure 4. Test with a known solution: time convergence in $\mathrm{L}^{2}$ norm for the pressure, for various meshes.

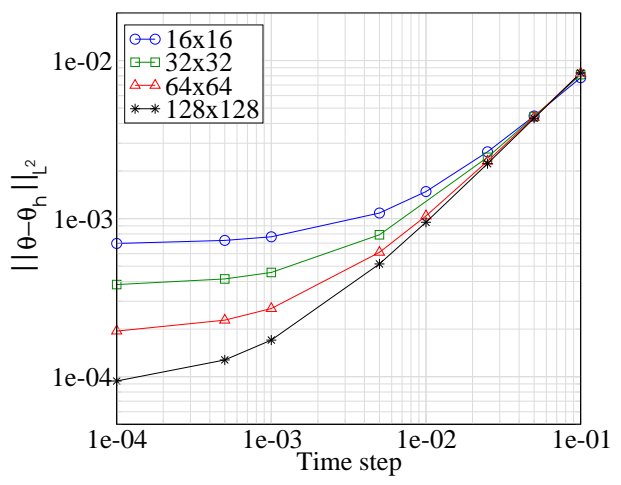

(a) Euler.

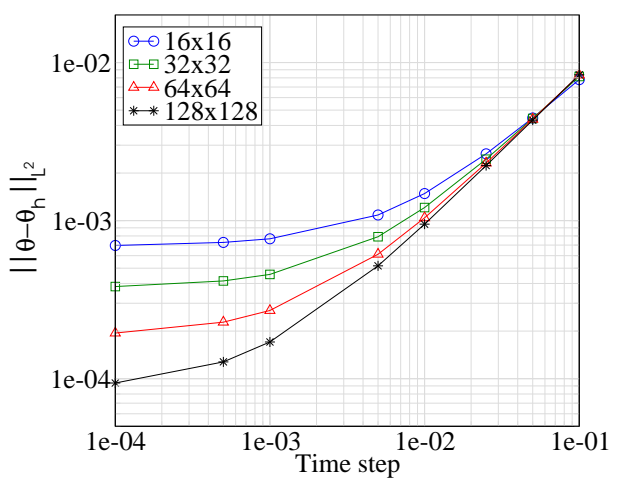

(b) Crank-Nicolson-like.

Figure 5. Test with a known solution: time convergence in discrete $\mathrm{L}^{2}$ norm for $\theta$, for various meshes.

$\theta$, in the following discrete $\mathrm{L}^{2}$ norm:

$$
\|\theta\|_{\mathrm{L}^{2}}=\left(\sum_{K \in \mathcal{M}}|K| \theta_{K}^{2}\right)^{\frac{1}{2}},
$$

which is usual in the finite volume framework. 


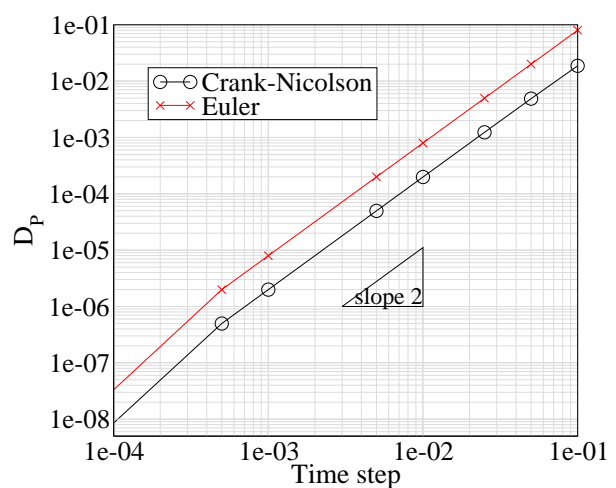

(a) Pressure gradient part.

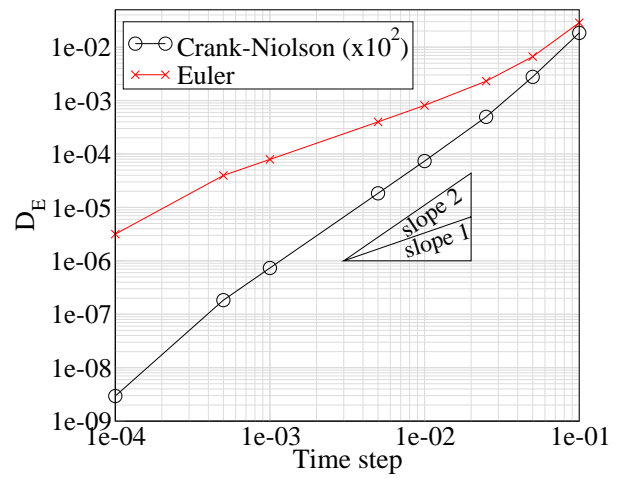

(b) Kinetic energy part.

Figure 6. Test with a known solution: magnitude of the two parts of the dissipation defect as a function of the time step for the Crank-Nicolson and the Euler time discretizations.

For each unknown, we observe a first order in time convergence, until reaching a plateau, the value of which corresponds to the space accuracy of the method. These figures confirm in particular the convergence properties of the overall algorithm even though we do not provide any convergence result in this paper.

We now investigate the behaviour of the dissipation defect for both the Euler and the CrankNicolson schemes. For this study, the mesh is fixed and chosen to be the $128 \times 128$ regular grid. In the definition of the dissipation defect given by (25) for Crank-Nicolson and by (30) for Euler, we distinguish the pressure gradient part, noted $\mathcal{D}_{P}^{n}$, from the kinetic energy part, noted $\mathcal{D}_{E}^{n}$, and we define $\mathcal{D}_{P}$ and $\mathcal{D}_{E}$ as the the maximum values in time of each part:

$$
\mathcal{D}_{P}=\max _{0 \leq n \leq N-1}\left|\sum_{k=0}^{n} \mathcal{D}_{P}^{k+1}\right|, \quad \mathcal{D}_{E}=\max _{0 \leq n \leq N-1}\left|\sum_{k=0}^{n} \mathcal{D}_{E}^{k+1}\right| .
$$

We draw on Figure 6 the obtained results as a function of the time step for both algorithms. As predicted by the analysis of Section 4.4, the pressure gradient part is of order two for both schemes and the kinetic energy part is of order two for the Crank-Nicolson scheme while the order is one for the Euler discretization. Moreover, it is also observed that the Crank-Nicolson scheme leads to a much smaller kinetic energy part (note that, for this scheme, the dissipation defect is multiplied by $10^{2}$ in the figure).

\section{2. $2 D$ laminar flow around a cylinder}

We address in this section some test cases which are derived from a benchmark referred to as the 2D-2 case in [30]. The geometry is sketched on Figure 7. A disk of diameter $D=0.1$ is located inside a 2D channel (the axis of the cylinder lying slightly below the centerline of the channel) and the fluid enters the domain on the left boundary, with an imposed parabolic velocity profile:

$$
u_{x}(0, y)=4 u_{m} y \frac{H-y}{H^{2}}, \quad u_{y}(0, y)=0,
$$

where $H=0.41 \mathrm{~m}$ is the height of the channel and $u_{m}=1.5$. The velocity is prescribed to zero at the other boundaries except for the right-hand side, where the flow leaves the domain; in our computation, we impose on this latter boundary an homogeneous Neumann boundary condition.

Our study is split into two parts. In the first step, the flow is supposed to be isotherm; the density $\rho$ is then constant, the flow is incompressible, and we can compare our results to the reference ones provided in [30]. In the second case, density variations are created in the flow by prescribing at the cylinder boundary a temperature greater than in the bulk.

The coarse mesh (mesh\#1) used for the presented computation is given on Figure 8; other meshes are refined with respect to this one, by setting to smaller and smaller values the discretization step 


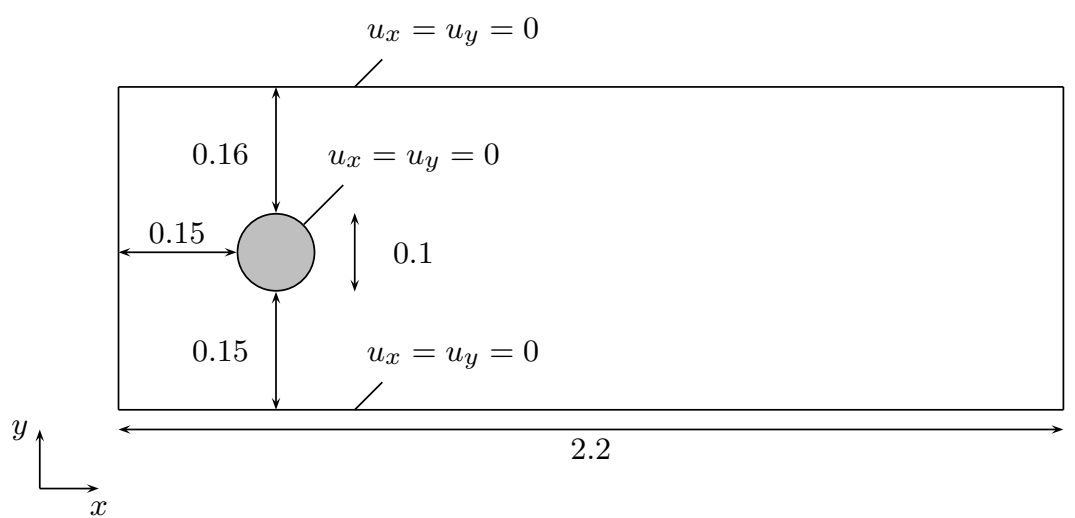

Figure 7. Laminar 2D flow around a cylinder - Geometry.

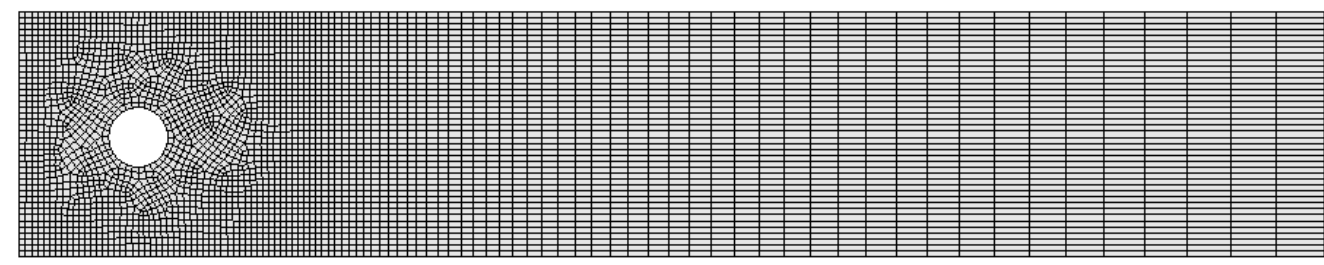

Figure 8. The coarse mesh (mesh\#1).

Table II. Laminar 2D flow around a cylinder - Description of the meshes used.

\begin{tabular}{|c|ccccc|}
\hline Computation & mesh\#1 & mesh\#2 & mesh\#3 & mesh\#4 & mesh\#5 \\
\hline Number of cells & 4033 & 12913 & 43009 & 76091 & 106101 \\
Space unknowns & 12256 & 39014 & 129527 & 228937 & 409099 \\
\hline
\end{tabular}

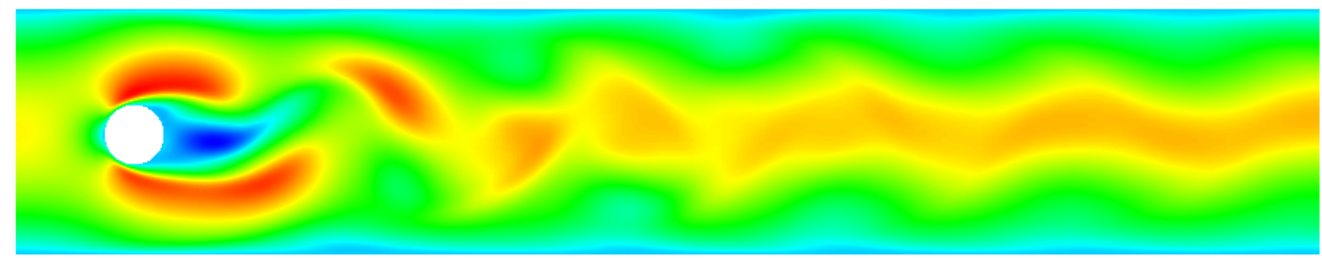

Figure 9. Laminar 2D flow around a cylinder - First component ( $x$-component) of the velocity.

along some characteristic lines (the boundaries and concentric circles around the cylinder). The numbers of cells and unknowns for each mesh are reported in Table II.

The flow is unsteady (see Figure 9 for a visualization at a given time). The characteristic flow quantities quoted in [30] are the maximum drag coefficient $c_{D \max }$, the maximum lift coefficient $c_{L_{\max }}$, the Strouhal number St and an instantaneous pressure difference $\Delta P$ between the front and end points of the cylinder, i.e. the points $(0.15,0.20)$ and $(0.25,0.20)$ (see [30, section 2.2]). Drag and lift coefficients are defined as follows. First, the drag and lift forces are expressed as:

$$
F_{D}=\int_{S}\left(\rho \nu \frac{\partial u_{t}}{\partial \boldsymbol{n}} \boldsymbol{n}_{y}-p \boldsymbol{n}_{x}\right) d S, \quad F_{L}=-\int_{S}\left(\rho \nu \frac{\partial u_{t}}{\partial \boldsymbol{n}} \boldsymbol{n}_{x}+p \boldsymbol{n}_{y}\right) d S
$$


Table III. Laminar 2D flow around a cylinder, isothermal case - Euler time discretization.

\begin{tabular}{|c|c|c|c|c|}
\hline Computation & $c_{D \max }$ & $c_{L \max }$ & $\mathrm{St}$ & $\Delta P$ \\
\hline mesh\#1 & 3.62 & 0.75 & 0.269 & 2.36 \\
mesh\#2 & 3.40 & 0.92 & 0.288 & 2.45 \\
mesh\#3 & 3.25 & 0.97 & 0.294 & $\mathbf{2 . 4 9}$ \\
mesh\#4 & $\mathbf{3 . 2 3}$ & 0.98 & 0.294 & $\mathbf{2 . 4 8 4}$ \\
mesh\#5 & $\mathbf{3 . 2 2}$ & $\mathbf{1 . 0 0}$ & 0.294 & $\mathbf{2 . 4 7 8}$ \\
\hline Reference range [30] & $3.22-3.24$ & $0.99-1.01$ & $0.295-0.305$ & $2.46-2.50$ \\
\hline
\end{tabular}

Table IV. Laminar 2D flow around a cylinder, isothermal case - Crank-Nicolson time discretization.

\begin{tabular}{|c|c|c|c|c|}
\hline Computation & $c_{D \max }$ & $c_{L \max }$ & $\mathrm{St}$ & $\Delta P$ \\
\hline mesh\#1 & 3.66 & 0.79 & 0.270 & 2.30 \\
mesh\#2 & 3.41 & 0.95 & 0.294 & 2.45 \\
mesh\#3 & 3.25 & 0.98 & $\mathbf{0 . 3 0 3}$ & $\mathbf{2 . 5 0}$ \\
mesh\#4 & $\mathbf{3 . 2 3}$ & $\mathbf{1 . 0 0}$ & $\mathbf{0 . 3 0 3}$ & $\mathbf{2 . 4 9}$ \\
mesh\#5 & $\mathbf{3 . 2 2}$ & $\mathbf{1 . 0 1}$ & $\mathbf{0 . 3 0 3}$ & $\mathbf{2 . 4 8}$ \\
\hline Reference range [30] & $3.22-3.24$ & $0.99-1.01$ & $0.295-0.305$ & $2.46-2.50$ \\
\hline
\end{tabular}

where $S$ stands for the boundary of the cylinder and $u_{t}$ for the tangential component of the velocity (namely $u_{t}=\boldsymbol{u} \cdot \boldsymbol{t}$ with $\boldsymbol{t}$ the tangential vector to $S$ ). Then, $c_{D}$ and $c_{L}$ are obtained by scaling $F_{D}$ and $F_{L}$ by $2 /\left(\rho \bar{u}^{2} D\right)$. The Strouhal number is $\mathrm{St}=D f / \bar{u}, f$ being the frequency of separation, evaluated from the temporal evolution of the lift coefficient.

Isothermal case The density is $\rho=1$ and the viscosity is $\mu=0.001$, so that the Reynolds number, defined as $\operatorname{Re}=\rho \bar{u} D / \mu$, where $\bar{u}=2 u_{x}(0, H / 2) / 3=1$, is equal to 100 . The time step varies from $10^{-3}$ for the coarsest mesh to $210^{-4}$ for the finest mesh, in order to keep a constant CFL number.

The computed values $\left(c_{D \max }, c_{L_{\max }}\right.$, St and $\left.\Delta P\right)$ are gathered in Tables III (Euler scheme) and IV (Crank-Nicolson scheme), together with the plausible range for the results derived from the set of contributions to the benchmark. Values entering this reference interval are typeset in bold. For the finest mesh, both schemes give results included in the benchmark reference range, except for the Strouhal number which is underestimated by the Euler scheme; in addition, with coarser meshes, the Crank-Nicolson scheme is clearly more accurate.

Anisothermal case The cylinder is now heated at a temperature $\theta_{h}$. The fluid enters in the domain with a lower temperature $\theta_{c}$ and the temperature is also fixed at $\theta_{c}$ at the top and bottom boundaries. We characterize the deviation from the isothermal case by the parameter $\varepsilon$ defined by

$$
\varepsilon=\frac{\theta_{h}-\theta_{c}}{\theta_{h}+\theta_{c}} .
$$

The Prandtl number is equal to 0.7 , the viscosity is fixed to 0.001 and the specific heat to 1000 . The density is given by the following law:

$$
\rho=\rho_{0} \frac{\theta_{c}}{\theta},
$$

with $\rho_{0}=1$. The parameter $\varepsilon$ varies in our tests from $\varepsilon=0$ (isothermal case) to $\varepsilon=0.8$, and thus the ratio $\rho_{0} / \varrho\left(\theta_{h}\right)$ varies from 1 (isothermal case) to $9(\varepsilon=0.8)$, since this ratio is equal to $(\varepsilon+1) /(\varepsilon-1)$.

Since the mesh here is unstructured, the SUSHI scheme is used for the approximation of the Laplace operator (see Remark 4.5) and the MUSCL scheme for the convective term [26] in the 


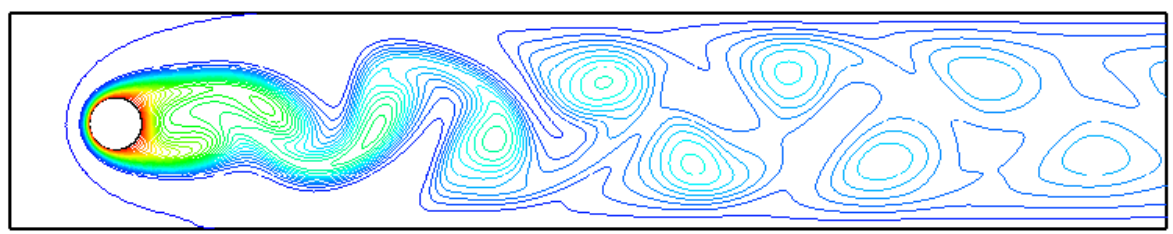

(a) $\varepsilon=0.4$

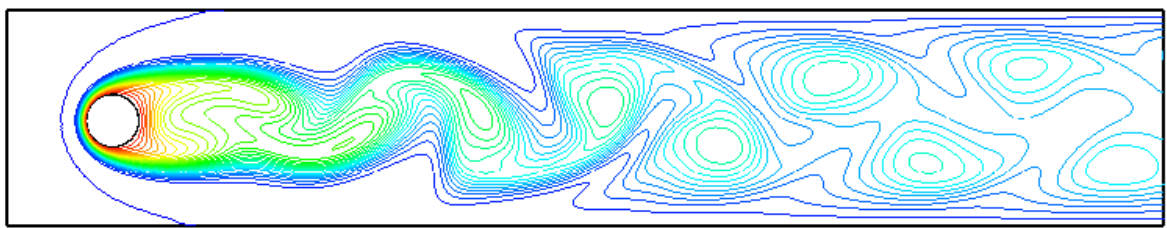

(b) $\varepsilon=0.6$

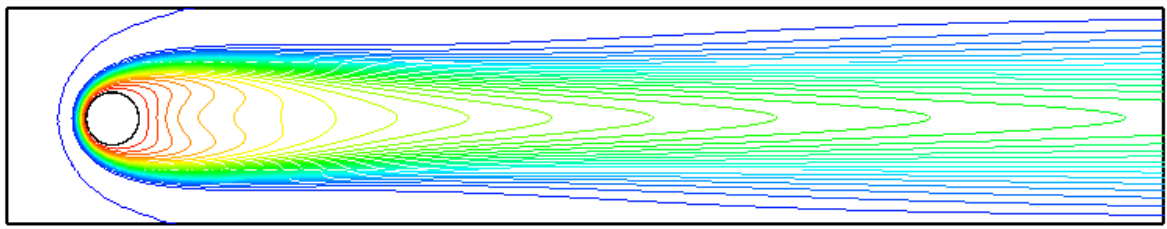

(c) $\varepsilon=0.8$

Figure 10. Laminar flow around a heated cylinder - Density field isolines for several $\varepsilon$ at time $t=10$.

Table V. Laminar flow around a heated cylinder - Drag and lift coefficients, Strouhal number and $\Delta P$ for several $\varepsilon$.

\begin{tabular}{|c|c|c|c|c|}
\hline Computation & $c_{D \max }$ & $c_{L \max }$ & St & $\Delta P$ \\
\hline isotherm & 3.22 & 1 & 0.303 & 2.48 \\
$\varepsilon=0.4$ & 3.54 & 0.56 & 0.316 & 2.59 \\
$\varepsilon=0.6$ & 3.84 & 0.236 & 0.342 & 2.71 \\
$\varepsilon=0.8$ & 4.62 & 0.0055 & steady & 3.04 \\
\hline
\end{tabular}

balance equation for $\theta$. The (constant) time step satisfies a CFL condition required by the MUSCL scheme and so varies between $10^{-4}$ and $210^{-5}$ in the computations.

On Figure 10, the density field is represented for $\varepsilon=0.4,0.6,0.8$. For small values of $\varepsilon$, as for the isothermal case, the flow is unsteady and oscillations appear in the wake of the cylinder. For $\varepsilon=0.4$, the amplitudes seem to be greater than for $\varepsilon=0.6$. This behaviour is coherent with an increase of Strouhal number when $\varepsilon$ increases (Table V). For $\varepsilon=0.8$, contrary to the other computations, the flow is steady and no oscillation appears. Heating the cylinder leads to a depressurization behind the cylinder which increases with $\varepsilon$ (Table V). The drag coefficient also increases with $\varepsilon$, whereas the lift coefficient decreases. For the steady case $(\varepsilon=0.8)$, the lift coefficient is very small and its value is similar to the reference value given in the benchmark [30] for the 2D steady case. 


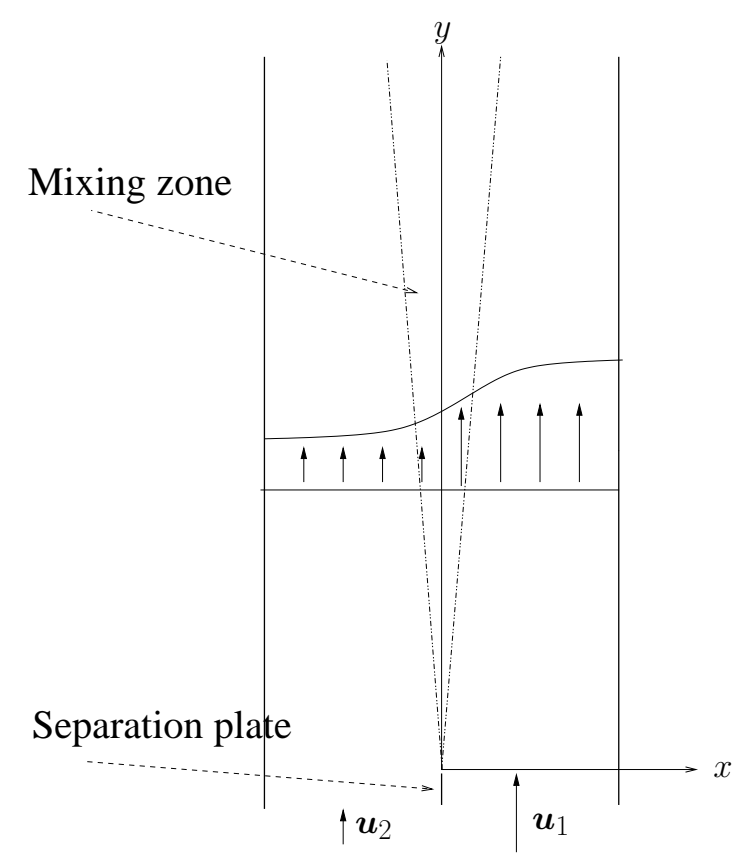

Figure 11. Turbulent mixing layer - Numerical set-up (front view).

\subsection{D turbulent mixing layer}

In this section, we address the simulation of an experimental study of a turbulent mixing layer in a vertical channel, described in [29]. The channel is divided at its bottom in two equal parts by a vertical plate, and water is injected in both parts with a different velocity. The mean velocity in the right side of the channel, $\boldsymbol{u}_{1}=0.61 \mathrm{~m} . \mathrm{s}^{-1}$, is greater than in the left one $\boldsymbol{u}_{2}=0.26 \mathrm{~m} . \mathrm{s}^{-1}$; a mixing layer, the thickness of which increases along the flow, is thus generated at the center of the channel (see Figure 11). The flow is incompressible and the density and the viscosity are respectively fixed at $\rho=10^{3} \mathrm{~kg} . \mathrm{m}^{-3}$ and $\mu=10^{-3}$ Pa.s.

Large Eddy Simulation is used to describe this experiment, and the subgrid scale eddy viscosity is given by the Smagorinsky model, with $C_{s}=0.12$ [20].

The computational domain is $\Omega=[-0.2 ; 0.2] \times[-0.01 ; 1.20] \times[-0.2 ; 0.2]$ (lengthes in meters), and the top of the plate is taken at $y=0$. Slip boundary conditions are prescribed on the lateral planes of $\Omega$ and outlet boundary conditions on the upper plane $y=1.20$. The separation plate is not taken into account in the simulation and mean experimental velocity profiles are imposed on the bottom plane $y=-0.01$. To generate the turbulent inflow conditions, the vortex method [16] is used.

The mesh is an uniform grid of $90 \times 90 \times 270$ cells and simulations are carried out with two time steps, $\delta t=10^{-2} \mathrm{~s}$ and $\delta t=10^{-3} \mathrm{~s}$, over the time interval [0s, 20s].

We compare the solutions given by the Crank-Nicolson-like pressure-correction scheme introduced here to the usual first order Euler pressure-correction scheme. Representations of the vorticity norm isosurfaces for the isovalue 6 are given on Figure 12 for several time steps. For the time step $\delta t=10^{-2} \mathrm{~s}$, Figure 12 shows that vortices present more complex structures with the Crank-Nicolson-like scheme (in other words, turbulent vortices are over-damped by the numerical diffusion generated by the first order Euler discretization). For $\delta t=10^{-3} \mathrm{~s}$, both schemes give similar results.

In the experiment, the (time-averaged) mean and root mean square (rms) axial velocity profiles are measured along the $x$-direction at several heights [29]. In our computations, we evaluate these quantities by averaging over a time-interval $\left[T=8 \mathrm{~s} ; T^{\prime}=20 \mathrm{~s}\right]$ for which the fully turbulent state is reached. Let $L$ be the integer number such that $T^{\prime}=T+L \delta t$. On a cell $K$, the mean value of a 


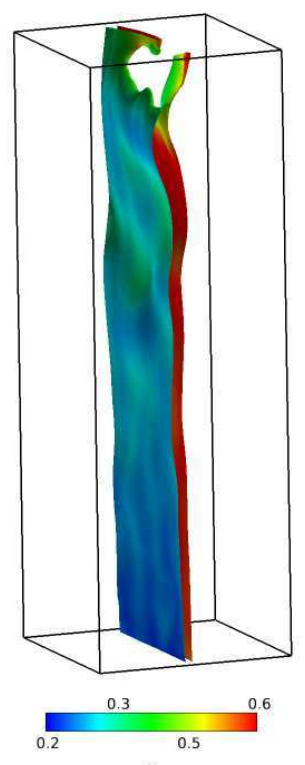

vy

(a) Euler, $\delta t=10^{-2} \mathrm{~s}$.

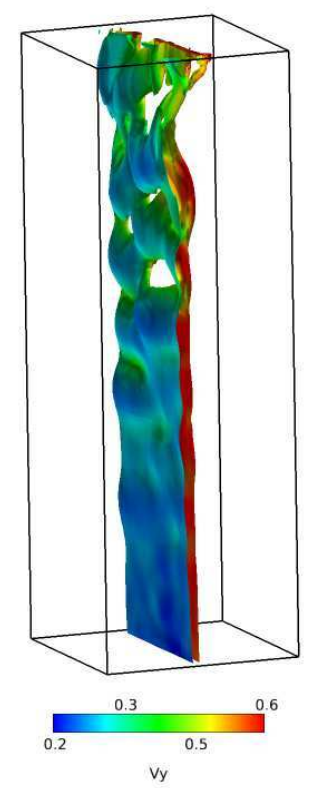

(c) Euler, $\delta t=10^{-3} \mathrm{~s}$.

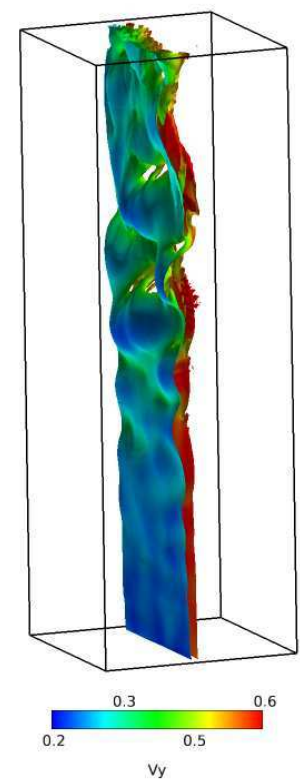

(b) Crank-Nicolson-like, $\delta t=10^{-2} \mathrm{~s}$.

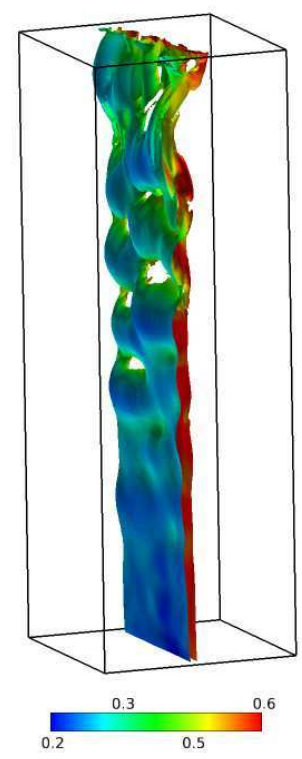

(d) Crank-Nicolson-like, $\delta t=10^{-3}$ s.

Figure 12. Turbulent mixing layer - Vorticity norm isosurface for the isovalue 6 at time 20s.

field $v$, denoted by $\langle v\rangle_{\mid K}$, is thus

$$
<v>_{\mid K}=\frac{1}{T^{\prime}-T} \sum_{j=0}^{L} \frac{\delta t}{|K|} \int_{K} v(\boldsymbol{x}, t+j \delta t) \mathrm{d} \boldsymbol{x},
$$

the mean axial velocity is given by $\left\langle\boldsymbol{u}_{y}\right\rangle_{\mid K}$ and the root mean square (rms) axial velocity by:

$$
\left(\boldsymbol{u}_{y}\right)_{r m s}=<\boldsymbol{u}_{y}^{2}>_{\mid K}-<\boldsymbol{u}_{y}>_{\mid K}^{2} .
$$

The comparison of experimental and numerical mean velocity profiles at $y=0.5$ (Figure 13) shows that the time convergence is achieved for $\delta t=10^{-2} \mathrm{~s}$ for both schemes and a good agreement is 


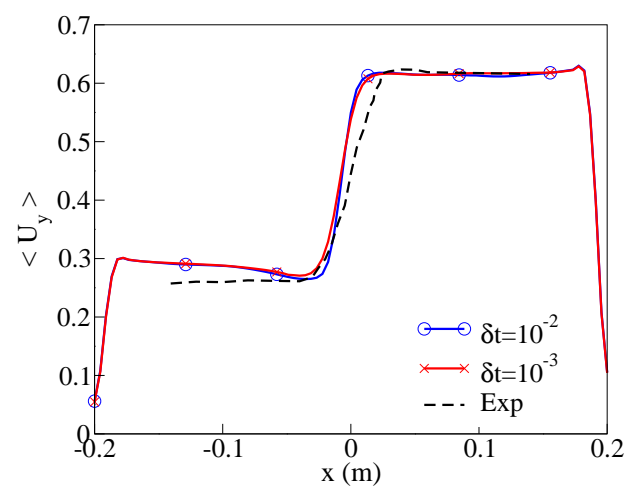

(a) Euler.

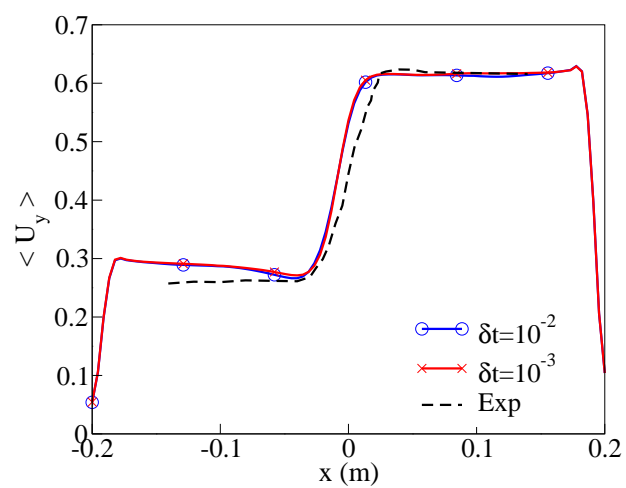

(b) Crank-Nicolson-like.

Figure 13. Turbulent mixing layer - Time averaged axial velocity at $y=0.5$ and $z=0$.

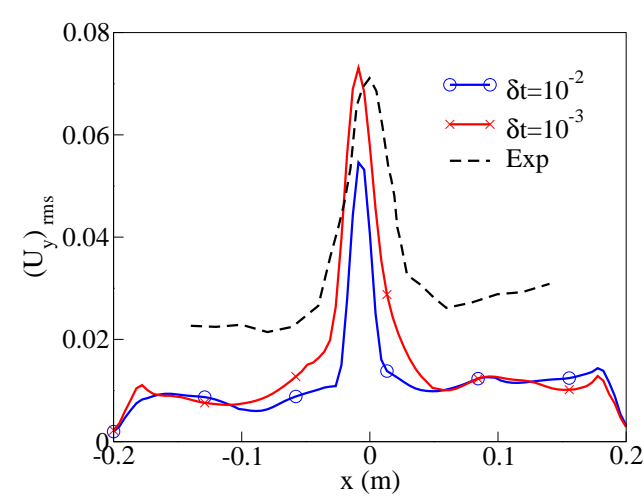

(a) Euler.

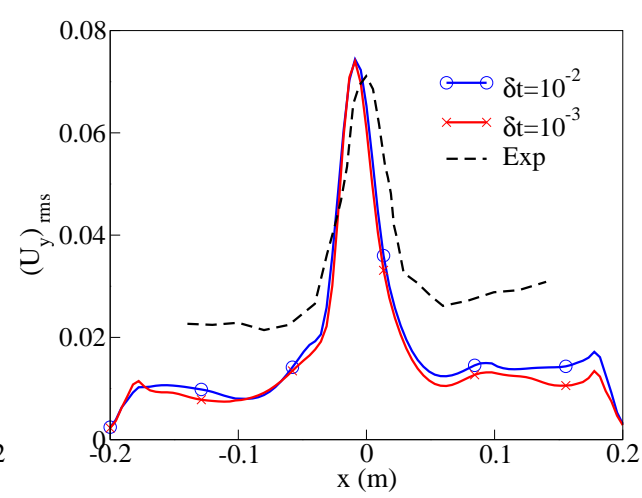

(b) Crank-Nicolson-like.

Figure 14. Turbulent mixing layer - Time rms axial velocity at $y=0.5$ and $z=0$.

observed with experimental results. Figure 14 shows the same profiles for the rms axial velocity. For this quantity, a difference is observed between both schemes at $\delta t=10^{-2} \mathrm{~s}$, the Crank-Nicolsonlike algorithm reproducing correctly the experimental peak, contrary to Euler. For $\delta t=10^{-3} \mathrm{~s}$, both schemes seem to have achieved convergence in time and, once again, results are in reasonable agreement with the experimental data.

\section{REFERENCES}

1. G. Ansanay-Alex, F. Babik, J.-C. Latché, and D. Vola. An L2-Stable Approximation of the Navier-Stokes Convection Operator for Low-Order Non-Conforming Finite Elements. International Journal For Numerical Methods In Fluids, 66:555-580, 2011.

2. P. Beaudan and P. Moin. Numerical Experiments on the Flow Past a Circular Cylinder at Sub-Critical Reynolds Number. Technical Report, TF-62, Department of Mechanic Engineering, Stanford University, 1994.

3. F. Brezzi and M. Fortin. Mixed and Hybrid Finite Element Methods. Number 15 in Springer Series in Computational Mathematics. Springer-Verlag, 1991.

4. P. G. Ciarlet. Handbook of Numerical Analysis: Finite Elements Methods - Basic Error Estimates for Elliptic Problems. In P. Ciarlet and J.L. Lions, editors, Handbook of Numerical Analysis, Volume II. North Holland, 1991.

5. O. Desjardins, G. Blanquart, G. Balarac, and H. Pitsch. High Order Conservative Finite Difference Scheme for Variable Density Low Mach Number Turbulent Flows. Journal of Computational Physics, 227, 2008.

6. F. Ducros, F Laporte, T. Soulères, V. Guinot, P. Moinat, and B. Caruelle. High-Order Fluxes for Conservative SkewSymmetric-Like Schemes in Structured Meshes: Application to Compressible Flows. Journal of Computational Physics, 161(1):114-139, 2000.

7. R. Eymard, T. Gallouet, and R. Herbin. Discretization of Heterogeneous and Anisotropic Diffusion Problems on General Nonconforming Meshes SUSHI: a Scheme Using Stabilisation and Hybrid Interfaces 1. Numerical Methods for Partial Differential Equations, 30:1009 -1043, 2009. 
8. F.N. Felten and T.S. Lund. Kinetic Energy Conservation Issues Associated with the Collocated Mesh Scheme for Incompressible Flow. Journal of Computational Physics, 215:465-484, 2006.

9. L. Gastaldo, R. Herbin, W. Kheriji, C. Lapuerta, and J.-C. Latché. Staggered Discretizations, Pressure Correction Schemes and all Speed Barotropic Schemes. Finite Volumes for Complex Applications 6, Prague, 2:39 - 55, 2011.

10. L. Georges, G. Winckelmans, and P. Geuzaine. Improving Shock-Free Compressible RANS Solvers for LES on Unstructured Meshes. Journal of Computational Applied Mathematics, 215:419-428, 2008.

11. J.-L. Guermond and L. Quartapelle. A Projection FEM for Variable Density Incompressible Flows. Journal of Computational Physics, 165:167-188, 2000.

12. By F. Ham, K. Mattsson, and G. Iaccarino. Accurate and Stable Finite Volume Operators for Unstructured Flow Solvers. Techniques, pages 243-261, 2006.

13. F.H. Harlow and J.E. Welch. Numerical Calculations of Time Dependent Viscous Incompressible Flow of Fluid with a Free Surface. Physics of Fluids, 8:2182 - 2189, 1965.

14. R. Herbin, W. Kheriji, and J.-C. Latché. Staggered Schemes for All Speed Flows. ESAIM proceedings, 35:122 $150,2012$.

15. ISIS. A CFD computer code for the simulation of reactive turbulent flows. https://gforge.irsn.fr/gf/project/isis.

16. N. Jarrin, S. Benhamadouche, D. Laurence, and R. Prosser. A Synthetic-Eddy-Method for Generating Inflow Conditions for Large-Eddy Simulation. International Journal of Heat and Fluid Flow, 27:585-593, 2006.

17. B. Larrouturou. How to Preserve the Mass Fractions Positivity when Computing Compressible Multi-Component Flows. Journal of Computational Physics, 95:59-84, 1991.

18. D.K. Lilly. On the Computational Stability of Numerical Solutions of Time-Dependent Non-Linear Geophysical Fluid Dynamics Problems. Monthly Weather Review., 93:11-26, 1965.

19. A. Majda and J. Sethian. The Derivation and Numerical Solution of the Equations for Zero Mach Number Combustion. Combustion Science and Techniques, 42:185-205, 1985.

20. M. Milelli. A Numerical Analysis of Confined Turbulent Bubble Plumes. PhD thesis, Swiss Federal Institute of Technology, Zurich, 2002.

21. R. Mittal and P. Moin. Suitability of Upwind-Biased Finite Difference Schemes for Large-Eddy Simulation of Turbulent Flows. AIAA Journal, pages 1415-17, 1997.

22. Y. Morinishi. Skew-Symmetric Form of Convective Terms and Fully Conservative Finite Difference Schemes for Variable Density Low-Mach Number Flows. Journal of Computational Physics, 229:276-300, 2010.

23. Y. Morinishi, T. S. Lund, O. V. Vasilyev, and P. Moin. Fully Conservative Higher Order Finite Difference Schemes for Incompressible Flow. Journal of Computational Physics, 143:90-124, 1998.

24. F. Nicoud. Conservative High-Order Finite-Difference Schemes for Low-Mach Number Flows. Journal of Computational Physics, 158:71-97, 2000.

25. PELICANS. Collaborative development environment. https://gforge.irsn.fr/gf/project/pelicans.

26. L. Piar, F. Babik, R. Herbin, and J.-C. Latché. A Formally Second-Order Cell Centered Scheme for ConvectionDiffusion Equations on General Grids. International Journal for Numerical Methods in Fluids, published online DOI: $10.1002 /$ fld.3688.

27. R. Rannacher and S. Turek. Simple Nonconforming Quadrilateral Stokes Element. Numerical Methods for Partial Differential Equations, 8:97-111, 1992.

28. Rolf Rannacher. On Chorin's Projection Method for the Incompressible Navier-Stokes Equations. 1530:167-183, 1992. Proceedings of a Conference held in Oberwolfach, Germany, August 18-24, 1991.

29. V. Roig. Zone de Mélange d'Ecoulements Diphasiques à Bulles. PhD thesis, University of Toulouse, 1993.

30. M. Schäfer and S. Turek. Benchmark Computations of Laminar Flow Around a Cylinder. In E.H. Hirschel, editor, Flow Simulation with High-Performance Computers II, volume 52 of Notes on Numerical Fluid Mechanics, pages 547-566, 1996.

31. P. K. Subbareddy and G. V. Candler. A Fully Discrete, Kinetic Energy Consistent Finite-Volume Scheme for Compressible Flows. Journal of Computational Physics, 228:1347-1364, 2009.

32. O. Vasilyev. High Order Finite Difference Schemes on Non-Uniform Meshes with Good Conservation Properties. Journal of Computational Physics, 157(2):746-761, 2000.

33. R. W. C. P. Verstappen and A. E. P. Veldman. Symmetry-Preserving Discretization of Turbulent Flow. Journal of Computational Physics, 187:343-368, 2003. 